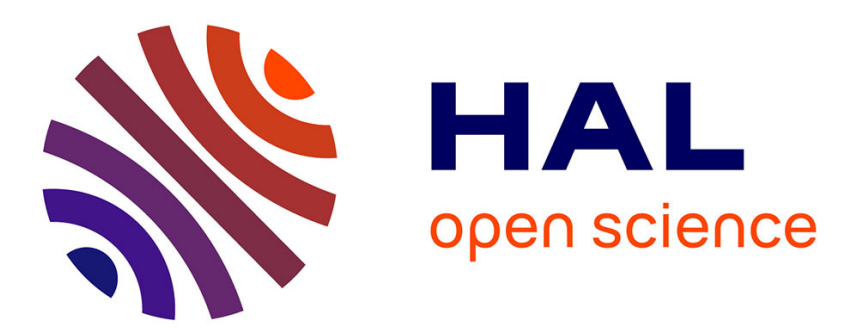

\title{
Évaluation du lambeau palmaire de Blauth lors de la prise en charge des syndactylies congénitales : à propos de 31 commissures
}

Margaux Delord

\section{- To cite this version:}

Margaux Delord. Évaluation du lambeau palmaire de Blauth lors de la prise en charge des syndactylies congénitales : à propos de 31 commissures. Médecine humaine et pathologie. 2018. dumas-01696868

\section{HAL Id: dumas-01696868 https://dumas.ccsd.cnrs.fr/dumas-01696868}

Submitted on 31 Jan 2018

HAL is a multi-disciplinary open access archive for the deposit and dissemination of scientific research documents, whether they are published or not. The documents may come from teaching and research institutions in France or abroad, or from public or private research centers.
L'archive ouverte pluridisciplinaire HAL, est destinée au dépôt et à la diffusion de documents scientifiques de niveau recherche, publiés ou non, émanant des établissements d'enseignement et de recherche français ou étrangers, des laboratoires publics ou privés. 


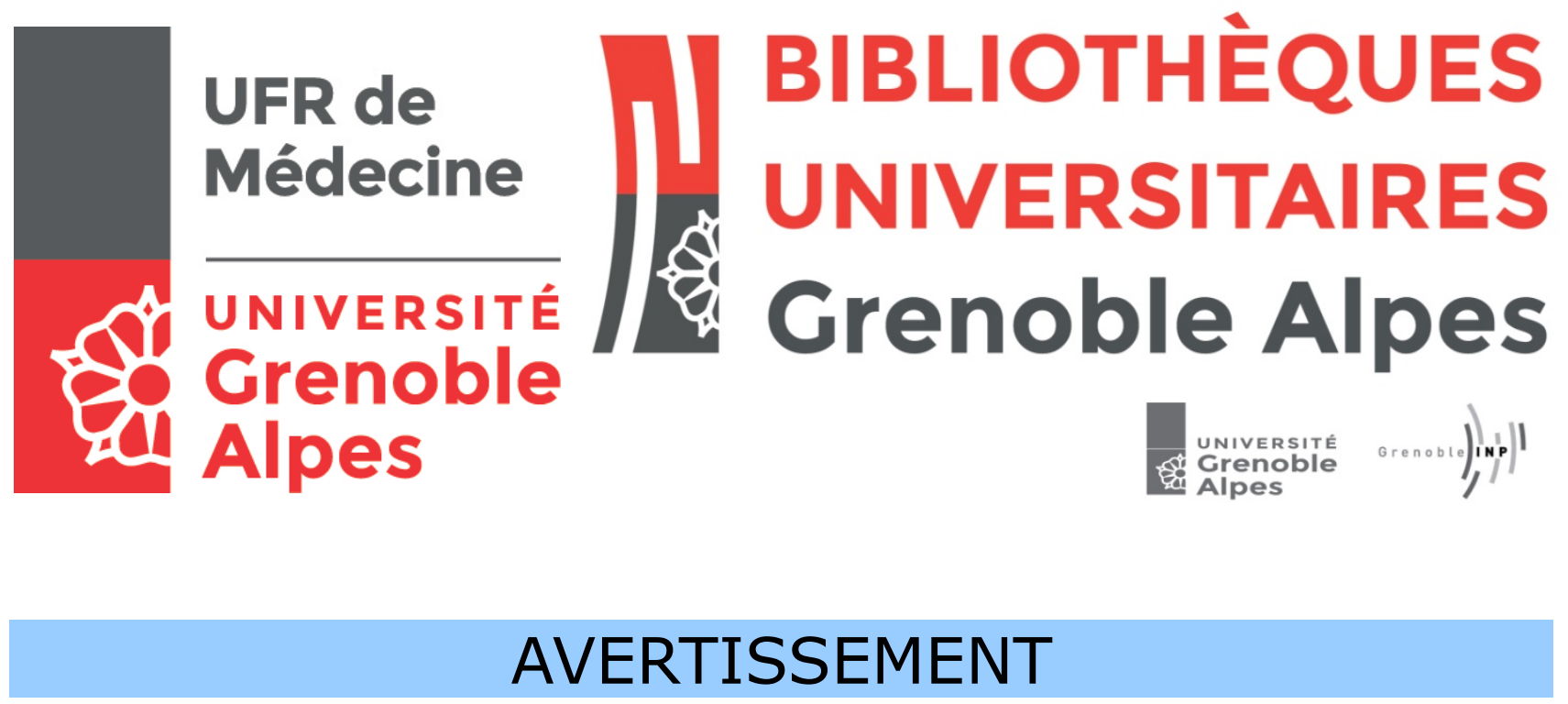

Ce document est le fruit d'un long travail approuvé par le jury de soutenance et mis à disposition de l'ensemble de la communauté universitaire élargie.

Il n'a pas été réévalué depuis la date de soutenance.

Il est soumis à la propriété intellectuelle de l'auteur. Ceci implique une obligation de citation et de référencement lors de I'utilisation de ce document.

D'autre part, toute contrefaçon, plagiat, reproduction illicite encourt une poursuite pénale.

Contact au SID de Grenoble : bump-theses@univ-grenoble-alpes.fr

\section{LIENS}

Code de la Propriété Intellectuelle. articles L 122. 4

Code de la Propriété Intellectuelle. articles L 335.2- L 335.10

http://www.cfcopies.com/juridique/droit-auteur http://www.culture.gouv.fr/culture/infos-pratiques/droits/protection.htm 
Année : 2018

\section{Évaluation du lambeau palmaire de Blauth lors de la prise en charge des syndactylies congénitales : à propos de 31 commissures}

THÈSE

PRESENTÉE POUR L’OBTENTION DU TITRE DE DOCTEUR EN MÉDECINE, DIPLÔME D'ÉTAT

Par Margaux DELORD

[Données à caractère personnel]

THÈSE SOUTENUE PUBLIQUEMENT A L'UFR DE MÉDECINE DE GRENOBLE

Le 24 janvier 2018

DEVANT LE JURY COMPOSÉ DE

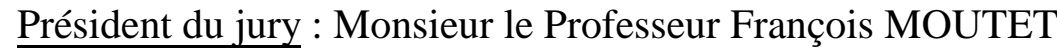

$\underline{\text { Membres : }}$

Madame le Docteur Alexandra FORLI, directrice de thèse

Monsieur le Professeur Laurent OBERT

Monsieur le Professeur Jacques GRIFFET

Monsieur le Docteur Denis CORCELLA

Monsieur le Docteur Jean Philippe GIOT 
Année 2017-2018

ENSEIGNANTS A L'UFR DE MEDECINE

\begin{tabular}{|c|c|c|}
\hline CORPS & NOM-PRENOM & Discipline universitaire \\
\hline PU-PH & ALBALADEJO Pierre & Anesthésiologie réanimation \\
\hline PU-PH & APTEL Florent & Ophtalmologie \\
\hline PU-PH & ARVIEUX-BARTHELEMY Catherine & Chirurgie générale \\
\hline PU-PH & BAILLET Athan & Rhumatologie \\
\hline PU-PH & BARONE-ROCHETTE Gilles & Cardiologie \\
\hline PU-PH & BAYAT Sam & Physiologie \\
\hline PU-PH & BENHAMOU Pierre Yves & Endocrinologie, diabète et maladies métaboliques \\
\hline PU-PH & BERGER François & Biologie cellulaire \\
\hline MCU-PH & BIDART-COUTTON Marie & Biologie cellulaire \\
\hline MCU-PH & BOISSET Sandrine & Agents infectieux \\
\hline PU-PH & BONAZ Bruno & Gastro-entérologie, hépatologie, addictologie \\
\hline PU-PH & BONNETERRE Vincent & Médecine et santé au travail \\
\hline PU-PH & BOREL Anne-Laure & Endocrinologie, diabète et maladies métaboliques \\
\hline PU-PH & BOSSON Jean-Luc & Biostatistiques, informatique médicale et technologies de communication \\
\hline MCU-PH & BOTTARI Serge & Biologie cellulaire \\
\hline PU-PH & BOUGEROL Thierry & Psychiatrie d'adultes \\
\hline PU-PH & BOUILLET Laurence & Médecine interne \\
\hline PU-PH & BOUZAT Pierre & Réanimation \\
\hline MCU-PH & BRENIER-PINCHART Marie Pierre & Parasitologie et mycologie \\
\hline PU-PH & BRICAULT Ivan & Radiologie et imagerie médicale \\
\hline PU-PH & BRICHON Pierre-Yves & Chirurgie thoracique et cardio- vasculaire \\
\hline MCU-PH & BRIOT Raphaël & Thérapeutique, médecine d'urgence \\
\hline MCU-PH & BROUILLET Sophie & Biologie et médecine du développement et de la reproduction \\
\hline PU-PH & CAHN Jean-Yves & Hématologie \\
\hline PU-PH & CARPENTIER Françoise & Thérapeutique, médecine d'urgence \\
\hline PU-PH & CARPENTIER Patrick & Chirurgie vasculaire, médecine vasculaire \\
\hline PU-PH & CESBRON Jean-Yves & Immunologie \\
\hline PU-PH & CHABARDES Stephan & Neurochirurgie \\
\hline PU-PH & CHABRE Olivier & Endocrinologie, diabète et maladies métaboliques \\
\hline PU-PH & CHAFFANJON Philippe & Anatomie \\
\hline PU-PH & CHARLES Julie & Dermatologie \\
\hline PU-PH & CHAVANON Olivier & Chirurgie thoracique et cardio- vasculaire \\
\hline PU-PH & CHIQUET Christophe & Ophtalmologie \\
\hline
\end{tabular}




\begin{tabular}{|c|c|c|}
\hline PU-PH & CHIRICA Mircea & Chirurgie générale \\
\hline PU-PH & CINQUIN Philippe & Biostatistiques, informatique médicale et technologies de communication \\
\hline MCU-PH & CLAVARINO Giovanna & Immunologie \\
\hline PU-PH & COHEN Olivier & Biostatistiques, informatique médicale et technologies de communication \\
\hline PU-PH & COURVOISIER Aurélien & Chirurgie infantile \\
\hline PU-PH & COUTURIER Pascal & Gériatrie et biologie du vieillissement \\
\hline PU-PH & CRACOWSKI Jean-Luc & Pharmacologie fondamentale, pharmacologie clinique \\
\hline PU-PH & CURE Hervé & Oncologie \\
\hline PU-PH & DEBILLON Thierry & Pédiatrie \\
\hline PU-PH & DECAENS Thomas & Gastro-entérologie, Hépatologie \\
\hline PU-PH & DEMATTEIS Maurice & Addictologie \\
\hline MCU-PH & DERANSART Colin & Physiologie \\
\hline PU-PH & DESCOTES Jean-Luc & Urologie \\
\hline MCU-PH & DETANTE Olivier & Neurologie \\
\hline MCU-PH & DIETERICH Klaus & Génétique et procréation \\
\hline MCU-PH & DOUTRELEAU Stéphane & Physiologie \\
\hline MCU-PH & DUMESTRE-PERARD Chantal & Immunologie \\
\hline PU-PH & EPAULARD Olivier & Maladies Infectieuses et Tropicales \\
\hline PU-PH & ESTEVE François & Biophysique et médecine nucléaire \\
\hline MCU-PH & EYSSERIC Hélène & Médecine légale et droit de la santé \\
\hline PU-PH & FAGRET Daniel & Biophysique et médecine nucléaire \\
\hline PU-PH & FAUCHERON Jean-Luc & Chirurgie générale \\
\hline MCU-PH & FAURE Julien & Biochimie et biologie moléculaire \\
\hline PU-PH & FERRETTI Gilbert & Radiologie et imagerie médicale \\
\hline PU-PH & FEUERSTEIN Claude & Physiologie \\
\hline PU-PH & FONTAINE Éric & Nutrition \\
\hline PU-PH & FRANCOIS Patrice & Epidémiologie, économie de la santé et prévention \\
\hline MCU-MG & GABOREAU Yoann & Médecine Générale \\
\hline PU-PH & GARBAN Frédéric & Hématologie, transfusion \\
\hline PU-PH & GAUDIN Philippe & Rhumatologie \\
\hline PU-PH & GAVAZZI Gaétan & Gériatrie et biologie du vieillissement \\
\hline PU-PH & GAY Emmanuel & Neurochirurgie \\
\hline MCU-PH & GILLOIS Pierre & Biostatistiques, informatique médicale et technologies de communication \\
\hline MCU-PH & GRAND Sylvie & Radiologie et imagerie médicale \\
\hline PU-PH & GRIFFET Jacques & Chirurgie infantile \\
\hline PU-PH & GUEBRE-EGZIABHER Fitsum & Néphrologie \\
\hline MCU-PH & GUZUN Rita & Endocrinologie, diabétologie, nutrition, éducation thérapeutique \\
\hline PU-PH & HAINAUT Pierre & Biochimie, biologie moléculaire \\
\hline PU-PH & HENNEBICQ Sylviane & Génétique et procréation \\
\hline PU-PH & HOFFMANN Pascale & Gynécologie obstétrique \\
\hline PU-PH & HOMMEL Marc & Neurologie \\
\hline PU-MG & IMBERT Patrick & Médecine Générale \\
\hline PU-PH & JOUK Pierre-Simon & Génétique \\
\hline PU-PH & JUVIN Robert & Rhumatologie \\
\hline
\end{tabular}




\begin{tabular}{|c|c|c|}
\hline PU-PH & KAHANE Philippe & Physiologie \\
\hline MCU-PH & KASTLER Adrian & Radiologie et imagerie médicale \\
\hline PU-PH & KRACK Paul & Neurologie \\
\hline PU-PH & KRAINIK Alexandre & Radiologie et imagerie médicale \\
\hline PU-PH & LABARERE José & Epidémiologie ; Eco. de la Santé \\
\hline MCU-PH & LABLANCHE Sandrine & Endocrinologie, diabète et maladies métaboliques \\
\hline MCU-PH & LANDELLE Caroline & Bactériologie - virologie \\
\hline MCU-PH & LAPORTE François & Biochimie et biologie moléculaire \\
\hline MCU-PH & LARDY Bernard & Biochimie et biologie moléculaire \\
\hline MCU-PH & LARRAT Sylvie & Bactériologie, virologie \\
\hline $\mathrm{MCU}-\mathrm{PH}$ & LE PISSART Audrey & Biochimie et biologie moléculaire \\
\hline PU-PH & LECCIA Marie-Thérèse & Dermato-vénéréologie \\
\hline PU-PH & LEROUX Dominique & Génétique \\
\hline PU-PH & LEROY Vincent & Gastro-entérologie, hépatologie, addictologie \\
\hline PU-PH & LEVY Patrick & Physiologie \\
\hline PU-PH & LONG Jean-Alexandre & Urologie \\
\hline PU-PH & MAGNE Jean-Luc & Chirurgie vasculaire \\
\hline MCU-PH & MAIGNAN Maxime & Thérapeutique, médecine d'urgence \\
\hline PU-PH & MAITRE Anne & Médecine et santé au travail \\
\hline MCU-PH & MALLARET Marie-Reine & Epidémiologie, économie de la santé et prévention \\
\hline MCU-PH & MARLU Raphaël & Hématologie, transfusion \\
\hline MCU-PH & MAUBON Danièle & Parasitologie et mycologie \\
\hline PU-PH & MAURIN Max & Bactériologie - virologie \\
\hline MCU-PH & MC LEER Anne & Cytologie et histologie \\
\hline PU-PH & MERLOZ Philippe & Chirurgie orthopédique et traumatologie \\
\hline PU-PH & MORAND Patrice & Bactériologie - virologie \\
\hline PU-PH & MOREAU-GAUDRY Alexandre & Biostatistiques, informatique médicale et technologies de communication \\
\hline PU-PH & MORO Elena & Neurologie \\
\hline PU-PH & MORO-SIBILOT Denis & Pneumologie \\
\hline PU-PH & MOUSSEAU Mireille & Cancérologie \\
\hline PU-PH & MOUTET François & Chirurgie plastique, reconstructrice et esthétique ; brûlologie \\
\hline MCU-PH & PACLET Marie-Hélène & Biochimie et biologie moléculaire \\
\hline PU-PH & PALOMBI Olivier & Anatomie \\
\hline PU-PH & PARK Sophie & Hémato - transfusion \\
\hline PU-PH & PASSAGGIA Jean-Guy & Anatomie \\
\hline PU-PH & PAYEN DE LA GARANDERIE Jean-François & Anesthésiologie réanimation \\
\hline MCU-PH & PAYSANT François & Médecine légale et droit de la santé \\
\hline MCU-PH & PELLETIER Laurent & Biologie cellulaire \\
\hline PU-PH & PELLOUX Hervé & Parasitologie et mycologie \\
\hline PU-PH & PEPIN Jean-Louis & Physiologie \\
\hline PU-PH & PERENNOU Dominique & Médecine physique et de réadaptation \\
\hline PU-PH & PERNOD Gilles & Médecine vasculaire \\
\hline PU-PH & PIOLAT Christian & Chirurgie infantile \\
\hline PU-PH & PISON Christophe & Pneumologie \\
\hline
\end{tabular}




\begin{tabular}{|c|c|c|}
\hline PU-PH & PLANTAZ Dominique & Pédiatrie \\
\hline PU-PH & POIGNARD Pascal & Virologie \\
\hline PU-PH & POLACK Benoît & Hématologie \\
\hline PU-PH & POLOSAN Mircea & Psychiatrie d'adultes \\
\hline PU-PH & PONS Jean-Claude & Gynécologie obstétrique \\
\hline PU-PH & RAMBEAUD Jacques & Urologie \\
\hline PU-PH & RAY Pierre & Biologie et médecine du développement et de la reproduction \\
\hline PU-PH & REYT Émile & Oto-rhino-laryngologie \\
\hline PU-PH & RIGHINI Christian & Oto-rhino-laryngologie \\
\hline PU-PH & ROMANET Jean Paul & Ophtalmologie \\
\hline PU-PH & ROSTAING Lionel & Néphrologie \\
\hline MCU-PH & ROUSTIT Matthieu & Pharmacologie fondamentale, pharmaco clinique, addictologie \\
\hline MCU-PH & ROUX-BUISSON Nathalie & Biochimie, toxicologie et pharmacologie \\
\hline MCU-PH & RUBIO Amandine & Pédiatrie \\
\hline PU-PH & SARAGAGLIA Dominique & Chirurgie orthopédique et traumatologie \\
\hline MCU-PH & SATRE Véronique & Génétique \\
\hline PU-PH & SAUDOU Frédéric & Biologie Cellulaire \\
\hline PU-PH & SCHMERBER Sébastien & Oto-rhino-laryngologie \\
\hline PU-PH & SCHWEBEL-CANALI Carole & Réanimation médicale \\
\hline PU-PH & SCOLAN Virginie & Médecine légale et droit de la santé \\
\hline MCU-PH & SEIGNEURIN Arnaud & Epidémiologie, économie de la santé et prévention \\
\hline PU-PH & STAHL Jean-Paul & Maladies infectieuses, maladies tropicales \\
\hline PU-PH & STANKE Françoise & Pharmacologie fondamentale \\
\hline MCU-PH & STASIA Marie-José & Biochimie et biologie moléculaire \\
\hline PU-PH & STURM Nathalie & Anatomie et cytologie pathologiques \\
\hline PU-PH & TAMISIER Renaud & Physiologie \\
\hline PU-PH & TERZI Nicolas & Réanimation \\
\hline MCU-PH & TOFFART Anne-Claire & Pneumologie \\
\hline PU-PH & TONETTI Jérôme & Chirurgie orthopédique et traumatologie \\
\hline PU-PH & TOUSSAINT Bertrand & Biochimie et biologie moléculaire \\
\hline PU-PH & VANZETTO Gérald & Cardiologie \\
\hline PU-PH & VUILLEZ Jean-Philippe & Biophysique et médecine nucléaire \\
\hline PU-PH & WEIL Georges & Epidémiologie, économie de la santé et prévention \\
\hline PU-PH & ZAOUI Philippe & Néphrologie \\
\hline PU-PH & ZARSKI Jean-Pierre & Gastro-entérologie, hépatologie, addictologie \\
\hline
\end{tabular}

PU-PH : Professeur des Universités et Praticiens Hospitaliers

MCU-PH : Maitre de Conférences des Universités et Praticiens Hospitaliers

PU-MG : Professeur des Universités de Médecine Générale

MCU-MG : Maître de Conférences des Universités de Médecine Générale 


\section{Remerciements :}

A Monsieur le Professeur François MOUTET,

Merci pour votre apprentissage rigoureux, dont j'ai eu le privilège de bénéficier durant mon externat puis mon internat. Vous me faites l'honneur de présider ce jury et je vous en remercie. Soyez assuré de mon admiration et de ma reconnaissance.

A Madame le Docteur Alexandra FORLI,

Merci pour tant de choses. Merci de m'avoir permis de travailler sur ce sujet et de m'avoir épaulé pour sa réalisation, merci pour le partage de ton savoir-faire, pour ton apprentissage pas à pas et avec patience, merci pour ta gentillesse et ta disponibilité. Ta rigueur force l'admiration et m'impressionnera toujours.

A Monsieur le Docteur Denis CORCELLA,

Merci pour les connaissances théoriques et pratiques que vous m'avez enseignées, pour vos conseils permettant de cibler l'essentiel. J'admire vos gestes rapides et précis dont aucun détail ne vient entailler la justesse. Et merci pour le côté humain que vous apportez aux relations professionnelles.

\section{A Monsieur le Professeur Laurent OBERT,}

Merci pour votre enseignement vif et dynamique dont j'ai pu profiter durant mon passage à Besançon. Je saurai faire bon usage du «Y-a-t-il quelque chose ? Y-a-t-il quelqu'un ?» comme vous me l'avez appris. Je ne garde de ma venue à Besançon que d'excellents souvenirs tant sur le plan chirurgical que humain. Et merci d'avoir fait le déplacement pour ma thèse, c'est un honneur.

A Monsieur le Professeur Jacques GRIFFET,

Merci d'avoir accepté de juger mon travail, soyez assuré de ma reconnaissance. Votre présence est un honneur.

A Monsieur le Docteur Jean-Philippe GIOT,

Merci pour votre enseignement chirurgical, médical et scientifique. Merci pour toute la patience que vous mettez pour nous apprendre à bien faire et merci de la confiance que vous m'accordez. 
A mes parents, merci pour votre soutien sans faille durant toutes ces années, merci pour l'amour que vous m'apportez.

A ma sista, merci d'avoir toujours été présente, notre complicité compte énormément pour moi.

A Romain, mon amour, mon p'tit fiancé merci de croire en moi depuis toujours, tu me rends plus forte (et plus cultivée, surtout en géographie :p). Merci de supporter ce rythme de travail et de $\mathrm{m}$ 'apporter une vie pleine de bonheurs et de rires.

A toute ma famille, merci,

Mon Papou et Mamie Hao : pour votre force de caractère dont j'espère avoir un peu hérité, à ma Manou et Papi Louis, partis trop tôt...

Ma marraine, mon parrain pour votre amour et soutien (et celui que vous portez au champagne et au vin :) )

Mes oncles et tantes, Tatie Dom', Maga et Tonton Bern' pour tous ces moments ensembles à Briançon ou autres...; Malou pour ces semaines de vacances dans le Sud à chanter du Céline Dion, Jean-Louis, Isa, Nadette, Tonton Michel...

Mes cousins, cousines : Max', Djoule, Mag', Alou comme une grande sœur, Jenn, Cora, Nico, Kikoune, Justine, Ly, Tara, Mai Hoa, vos moitiés, vos enfants et mon filleul Théo, pour tous ces précieux moments partagés en famille depuis toujours et ceux qui nous restent encore à passer.

A tous ces grands Docteurs chirurgiens de la main,

A Mick, plus qu'un maître, une sorte de grand frère, d'abord co-interne : tu as su me guider dans le droit chemin (celui de la chirurgie de la main ${ }^{\wedge} \wedge$ ), m'apprendre des bases pratiques avec patience. Je suis maintenant ton interne et je te remercie énormément pour tout le temps que tu consacres à mon enseignement tant théorique que pratique. Et même si râler c'est un peu ton super pouvoir, je resterai toujours admirative de tes connaissances et ton savoir-faire chirurgical. Merci pour tout.

A Billy, merci pour ces semestres d'internes passés ensembles, puis tes conseils tant chirurgicaux que personnels lors de ton assistanat. Ton calme est un exemple.

A Alessandro, merci pour ta disponibilité et ton enseignement durant mes années d'externe puis d'interne.

A Marion, j'apprécie beaucoup travailler avec toi, pas de stress, quand faut y aller, faut y aller. II me tarde de travailler à nouveau à tes côtés.

Ceux de Chirurgie Plastique et Maxillo : Marine, Emma, Juliette, Isé

Ceux rencontrés lors de mon semestre à Besançon : Damien pour ton RERC, François, Daniel, Isabelle, Pr Pauchot, Julien, Pierre-Bastien, Thomas, Séverin, Pauline, Grégoire, Marie. Merci pour votre accueil.

Ceux non encore cités qui m'ont formée : Dr Morand, Dr Chirpaz, Dr Jager, Dr lonescu, Dr Allamel, Dr Houillon, Dr Duprez, Dr Oulie, Dr Mestrallet, Dr Morati. 
A tous ces futurs grands Docteur,

Rodo, ami avant même de commencer l'internat, nous avons fait nos premiers pas d'internes ensemble. Ton parcours et ta détermination $m^{\prime}$ 'impressionnent et je suis sûre que tu deviendras un grand chirurgien. J'espère que notre amitié perdurera même si un jour nos chemins professionnels s'éloignent.

Clem, consciencieuse et organisée c'était un plaisir de partager un semestre avec toi, pas d'ennui possible tu as toujours une histoire à raconter. Et même si je ne partage pas cet inconditionnel amour pour les chats, j'espère que nous resterons proches.

Chacha, enfin co-interne, et c'est un plaisir.

Et tous les co-internes : Virginie, Nico Coste, Christol, Maximilien, Jean-Charles pour ta bonne humeur, Jérémy pour ton organisation, Pia, Sophie, Loric.

Les Docteurs et Internes que le travail a mis sur mon chemin et qui sont des amis: Oliv' alias Seuricate, Léo, Nico dit Bremondo pour ta gentillesse, Camille pour nos origines communes, Camille, Thomas, Sam', Flo, Gilles, Edouard, Vincent, Jessica, Gavard...

Et merci à toutes les équipes médicales et paramédicales avec lesquelles j'ai travaillé et je travaille, en particulier les équipes de chirurgie de la main et de chirurgie plastique et maxillo-faciale du $\mathrm{CHU}$ de Grenoble. Les secrétaires de chocs, les infirmières au top, les IBODES qui dépotent, la parfaite équipe d'Ergo-Kiné, les anesthésistes et IADES. Trop nombreux pour être tous cités, ma considération n'en ai pas moindre.

A mon noyau, et vos moitiés ma Rébec pour notre amitié si précieuse depuis plus de 15 ans, ma Margoune : ma sosie depuis des années, ma Nono Belgique, Suisse, peu importe la distance notre amitié reste forte, ma Lili une volonté de fer dans tout ce que tu entreprends, ma Clem'

Aux petits $V^{*}$ et vos moitiés: ma bledi love, mon p'tit grain de folie chéri, ma grinète pour nos premiers pas d'externe ensemble et notre amitié depuis la P2, ma Sarou pour ta joie de vivre en toutes circonstances, Lison la réservée au grand cœur, Rosa future grande neurologue, Agathe pour notre vocation commune, Blanket, Margounette again, pour toutes nos soirées, diners aux conversations diverses et variées (ou pas).

A tous les Meylanais, amis depuis toujours, merci de me faire sortir de mon monde hospitalier. Bess plus de 10 ans d'amitié, Claro ma toulousaine adorée, Daba la belle princesse du désert, Djodjo mon fils adoptif, Gnak pour ta gentillesse, Strazz la joyeuse, Jojo pour ton rire communicatif, Mathou Capi Chef toujours au top, Capel' le bringueur barbu , Zbik pour ta bonne ambiance communicative, Taf le chilien, Renoi la tornade, Toxi l'insta montagnarde, Naj' pour la blague de trop, Pej pour ton originalité et ton ouverture d'esprit, Toto le ministre je n'oublierai jamais ce TP de SVT, Tuk pour ton humour sans limites, Blanki, Hus, Jérem, Monti, Carpan, Mélie, Tourist, Marbar, Maxi. 


\section{SOMMAIRE}

Abréviations …………………………………………………………………... page 10

Résumé

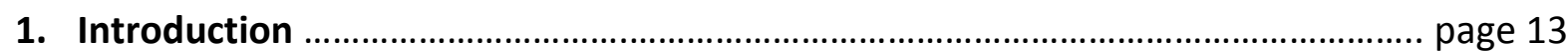

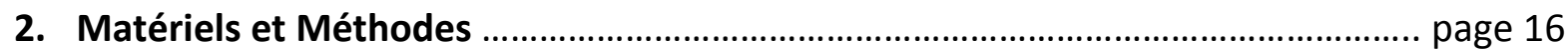

2-a. Population ................................................................................. page 16

2-b. Prise en charge chirurgicale ......................................................... page 19

2-c. Analyses statistiques ................................................................ page 23

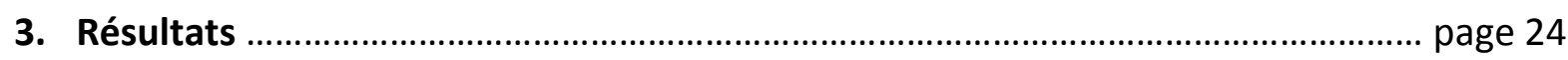

3-a. Population opérée .................................................................................. page 24

3-b. Population revue ........................................................................................ page 25

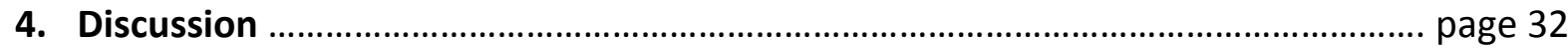

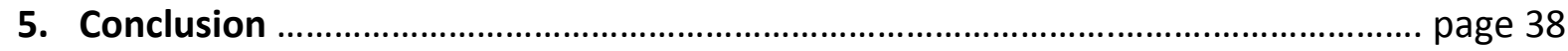

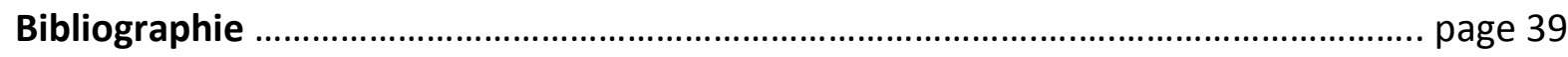

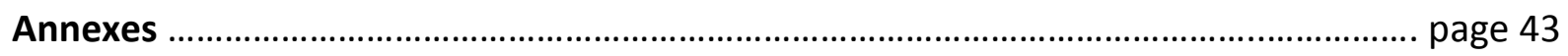

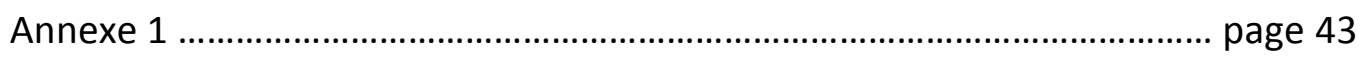

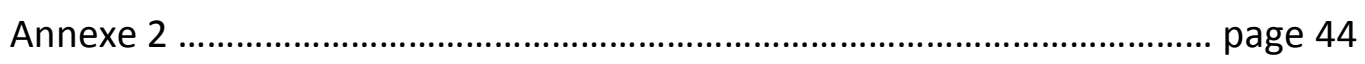




\section{Abréviations :}

BMRC: British medical research council

CHU: Centre Hospitalier Universitaire

GPM: Greffe de peau mince

GPT: Greffe de peau totale

IFSSH: International Federation of Societies for Surgery of the Hand

OMT: Oberg, Manske et Tonkin

USA: United States of America

VSS: Vancouver Scar Scale 


\section{Résumé}

Introduction : Les syndactylies congénitales résultent d'un défaut de différenciation tissulaire entrainant l'absence de séparation digitale au cours de l'embryogénèse. II s'agit d'une malformation fréquemment rencontrée dont la prise en charge chirurgicale est décrite depuis le XIXème siècle. Elles sont de sévérité variable et peuvent être isolées ou associées à d'autres anomalies dans le cadre de syndromes malformatifs. La construction de la néocommissure fait partie des éléments déterminants de la qualité du traitement chirurgical. L'objectif principal de notre étude était de quantifier la rétraction commissurale lors de la prise en charge des syndactylies congénitales opérées par un lambeau palmaire de Blauth.

Matériels et méthodes: Nous avons réalisé une étude monocentrique rétrospective. Nous avons inclus les enfants présentant au moins une syndactylie des doigts longs, simple ou complexe, syndromique ou non, opérés entre le $1^{\text {er }}$ janvier 1993 et le $1^{\text {er }}$ juin 2015 par une plastie commissurale palmaire selon la technique de Blauth. Pour compléter la couverture des faces latérales des doigts, tous les enfants bénéficiaient d'une greffe de peau totale prélevée au niveau du pli inguinal. Nous avons recueilli les données épidémiologiques et de prise en charge à partir des dossiers, puis nous avons évalué les enfants lors d'une consultation de révision. Notre critère de jugement principal était la rétraction commissurale évaluée selon l'échelle de Withey. Nos critères de jugements secondaires étaient: la qualité cicatricielle selon l'échelle de Vancouver, la présence d'une pilosité et/ou d'une dyschromie, la mesure de l'écartement digital, la présence d'un flessum digital, d'une clinodactylie, la mesure de la mobilité en flexion et en extension, la sensibilité de la pulpe et la satisfaction des parents et/ou de l'enfant.

Résultats : Soixante-cinq commissures ont été opérées avec un âge moyen lors de la chirurgie de 18,9 mois. Huit commissures ont nécessité une reprise chirurgicale.

Nous avons revu 20 patients soit 31 commissures, avec un recul moyen de 9 ans. Selon la classification de Withey, $58 \%$ des commissures étaient évaluées de grade $0,36 \%$ de grade 1 et $6 \%$ de grade 2 . Aucune commissure n'était quantifiée de grade 3 ou 4 . L'âge au moment de 
la chirurgie, le sexe, la commissure atteinte, le type de la syndactylie n'étaient pas corrélés à la présence d'une rétraction commissurale. Une diminution de la mobilité et la présence d'un flessum étaient plus souvent présentes dans les formes complexes. Quatre-vingt-sept pourcent des greffes de peau totale présentaient une dyschromie et $42 \%$ une pilosité. Sur le plan global, $93 \%$ des parents étaient satisfaits et 100\% des enfants en âge de répondre.

Conclusion : Le lambeau palmaire de Blauth est un lambeau fiable assurant de bons résultats fonctionnels et un taux de rétraction commissurale faible pour la reconstruction de la néocommissure. Sur le plan esthétique les plaintes principales concernent la dyschromie et la pilosité liées aux greffes de peau totale. 


\section{Introduction}

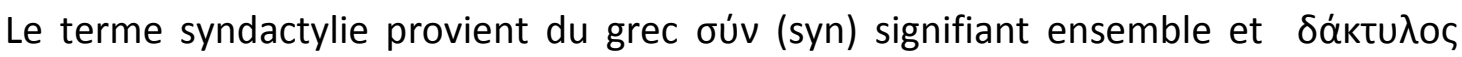
(dactulos) signifiant doigt. Dans les pays occidentaux, il s'agit de la malformation congénitale de la main la plus fréquente après l'hexadactylie et son incidence est d'environ 1/2000-2500 naissance (1). Par opposition aux syndactylies amniotiques dont le processus malformatif est différent, notre étude porte uniquement sur le sujet des syndactylies embryonnaires. C'est au $38^{\text {ème }}$ jour de vie embryonnaire que la plaque digitale commence à subir des processus d'apoptose permettant l'apparition des rayons digitaux (2). Dans sa forme isolée, une mutation du gène HOXD13 serait responsable d'un défaut du processus de mort cellulaire et donc d'une insuffisance de segmentation digitale $(3,4)$. Peu de facteurs de risques sont rapportés dans la littérature, mais le tabagisme durant la grossesse semble augmenter le risque de malformations digitales (5). Pour les formes familiales, il s'agit d'une transmission autosomique dominante à pénétrance variable.

Les syndactylies congénitales ont longtemps été classées dans le groupe II (défauts de différentiation) de la classification de Swanson (6) adoptée par l'International Federation of Societies for Surgery of the Hand (IFSSH) (7). Plus récemment, la classification Oberg, Manske et Tonkin (OMT) se basant sur les dernières données de biologie moléculaire du développement, les placent dans le groupe des malformations atteignant uniquement la main sans axe spécifique et atteignant les tissus mous (8). Cette classification distingue les syndactylies syndromiques comme une entité à part en les répertoriant dans le groupe " syndromes ». Cliniquement, il est différencié les formes simples ou membraneuses (atteinte du revêtement cutané seul) des formes complexes (fusions osseuses, rayons surnuméraires, brachyphalangie, anomalies tendineuses) ; et les formes complètes (atteinte de l'ensemble de la hauteur du doigt) des formes incomplètes. Le bilan clinique initial est toujours complété par la réalisation de radiographies de la main.

Les syndactylies sont plus fréquemment retrouvées chez le sexe masculin (ratio 3/2) et les caucasiens sont 10 fois plus atteints que les populations africaines ou asiatiques (9). Elles atteignent le plus souvent la $3^{\text {ème }}$ commissure, autant la main droite que la main gauche et l'atteinte peut être bilatérale (10). 
Afin d'améliorer la fonction, mais également pour des raisons esthétiques une prise en charge chirurgicale est proposée. Cependant, la mesure de la circonférence de 2 doigts accolés par rapport à la circonférence de 2 doigts séparés laisse présumer d'un déficit cutané d'environ 30\%. Concernant l'architecture d'une commissure native, elle comporte une face dorsale oblique qui descend progressivement en distale et en palmaire, formant un angle de $45^{\circ}$ par rapport au plan palmaire. Le bord libre de la commissure se situe à mi hauteur de la première phalange (11) (Fig. 1).

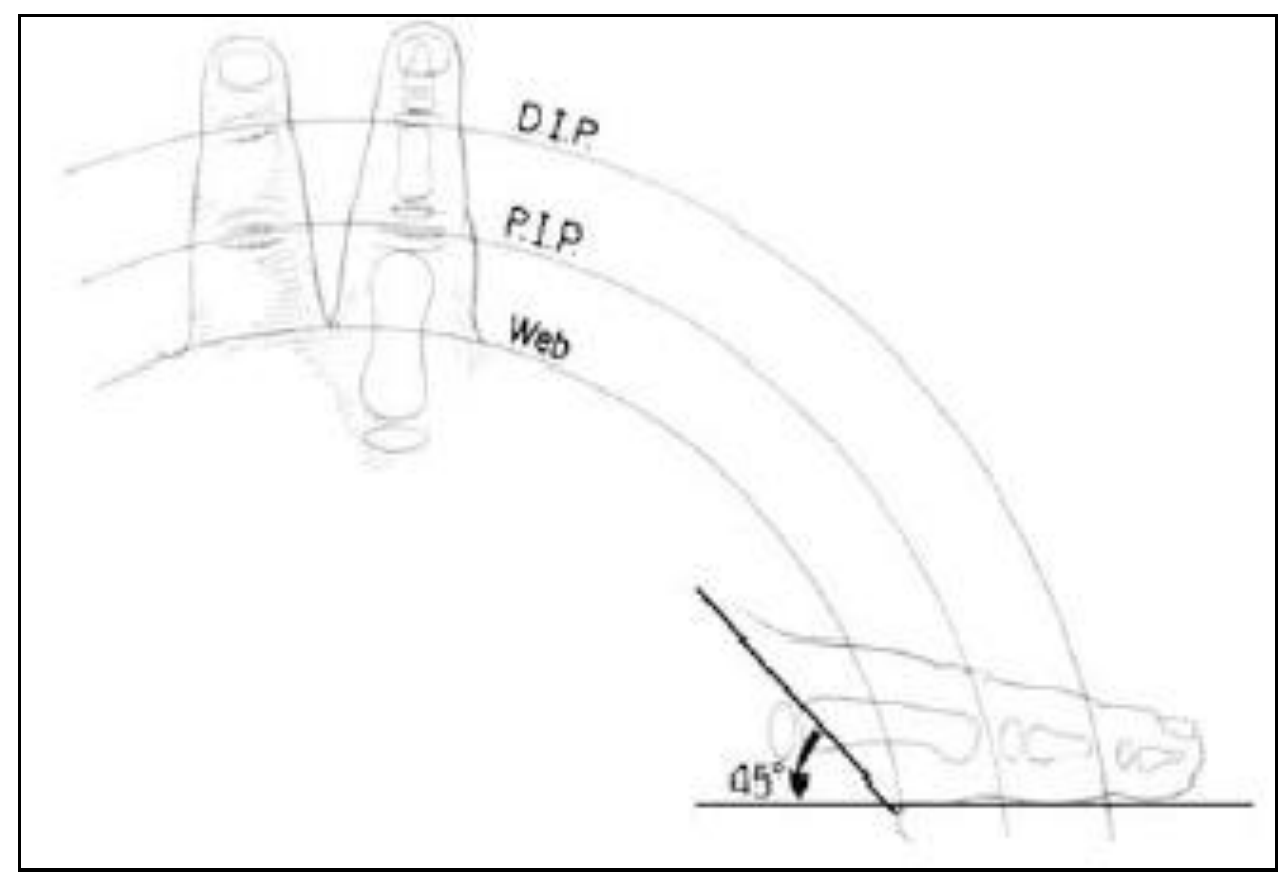

In: Mathes SJ, Hentz VR. Plastic surgery vol.8, Saunders Elsevier; Philadelphia 2006.

Figure 1 : Caractéristiques d'une commissure native.

Le traitement chirurgical se base donc sur la séparation digitale, la création d'une néocommissure et la gestion des faces latérales des doigts avec couverture des pertes de substances cutanées. C'est à partir du XIXème siècle que les premières publications concernant sa prise en charge apparaissent. En 1810, Zeller a décrit le premier lambeau dorsal triangulaire (12) pour la reconstruction de la commissure. Depuis, de nombreuses variantes ont été décrites, utilisant à la fois des lambeaux commissuraux dorsaux : lambeau en $T$, lambeau en oméga, lambeau rectangulaire ; parfois même croisés (lambeau dorsal et palmaire) : lambeau en $\mathrm{V}-\mathrm{Y}$, deux lambeaux triangulaires (13). 
En 1972, Blauth (14) a rapporté l'utilisation d'un lambeau palmaire en oméga. Toutes ces techniques ont deux objectifs communs : maximiser l'écartement digital et limiter la rétraction commissurale.

La rétraction commissurale représente une des principales complications à long terme du traitement chirurgical des syndactylies congénitales. Son taux varie de 0 à $48 \%(15,16)$ selon les études et cette grande variabilité peut être expliquée par l’hétérogénéité des séries.

L’objectif principal de notre étude était de quantifier la rétraction commissurale lors de la prise en charge chirurgicale des syndactylies congénitales opérées par un lambeau palmaire de Blauth. 


\section{Matériels et Méthodes}

\section{2-a. Population}

II s'agit d'une étude monocentrique rétrospective réalisée au CHU de Grenoble dans le service de chirurgie reconstructrice des membres et chirurgie de la main.

Nos critères d'inclusions étaient :

- patients présentant au moins une syndactylie congénitale embryonnaire des doigts longs, de forme simple ou complexe, syndromique ou non, complète ou incomplète,

- patients ayant bénéficié d'une plastie commissurale par un lambeau palmaire selon la technique de Blauth,

- $\quad$ patients opérés entre le $1^{\text {er }}$ janvier 1993 et le $1^{\text {er }}$ juin 2015.

Etaient exclus, les patients présentant une syndactylie dite acquise sur séquelles traumatiques ou de brûlures, les syndactylies amniotiques et les syndactylies de la $1^{\text {er }}$ commissure.

L'examinateur était toujours le même et ne faisait pas partie des différents opérateurs.

Les données épidémiologiques recueillies étaient : l'âge de l'enfant lors de la chirurgie, le sexe, le terme, la présence d'antécédents orientant vers une forme syndromique et d'antécédents familiaux de syndactylies.

Concernant la syndactylie, nous nous sommes intéressés à la commissure atteinte, le coté, sa forme simple ou complexe, la présence d'un appareil unguéal double ou commun. Les formes complexes étaient définies par la présence d'un rayon surnuméraire, l'existence d'une phalange delta, une brachyphalangie, la présence d'une fusion osseuse ou une anomalie tendineuse. A l'aide de la classification de Malek (17) (Fig. 2) les syndactylies étaient classées en formes complètes (A) ou incomplètes (B ou C). 


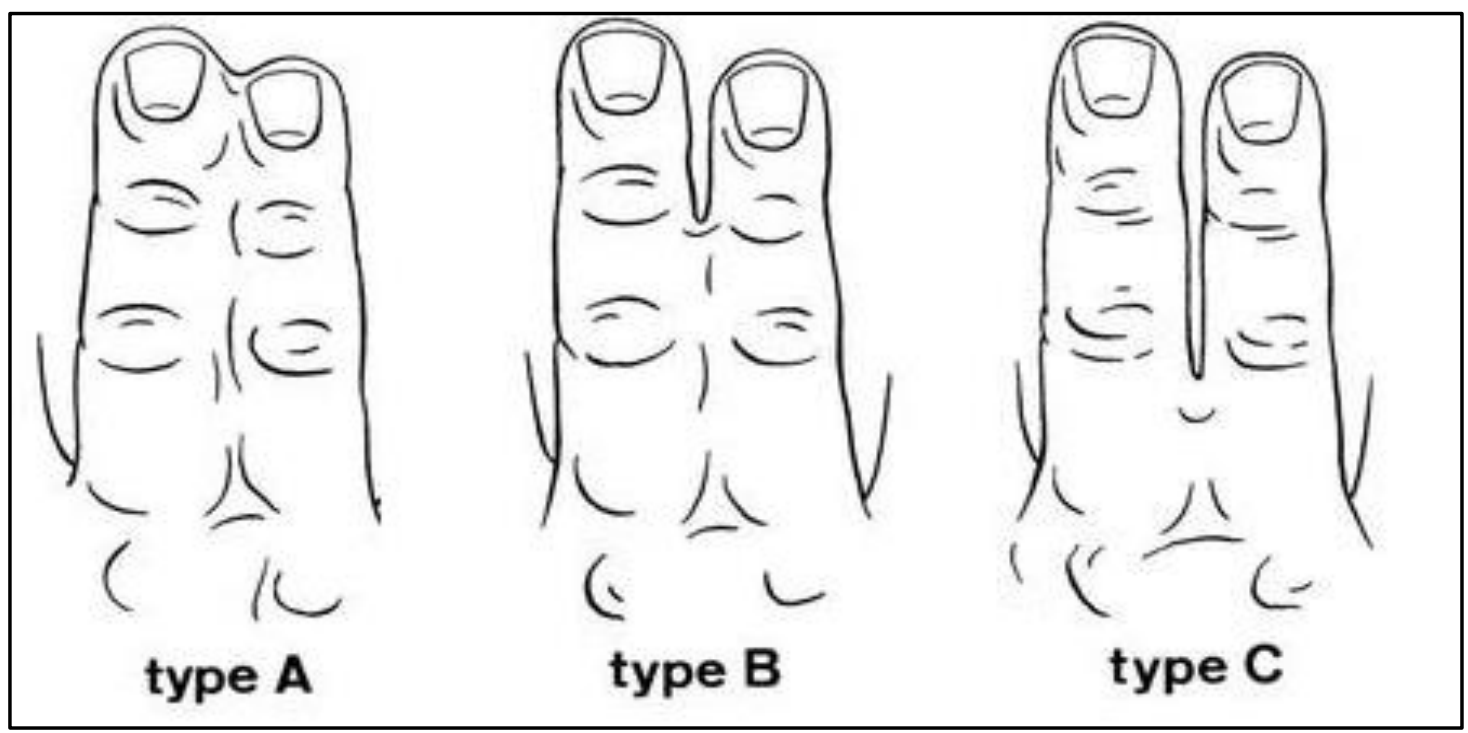

In Malek R. Syndactylies Congénitales. In : Encycl Med Chir. Paris: Appareil locomoteur; 1991.

Figure 2 : Classification de Malek.(17)

Concernant la prise en charge nous avons recueilli les données suivantes à partir des comptes rendus opératoires: l'âge de l'enfant lors de l'intervention, le type de greffe utilisé pour la couverture des faces latérales, la zone de prélèvement, la présence de complications peropératoires, la réalisation ou non d'un lambeau de Buck Gramcko, le type de pansement réalisé.

La durée totale des soins, l'utilisation de pressothérapie, la présence de complications post-opératoire ont également étaient relevées. En cas de reprise chirurgicale, nous avons recueilli le délai depuis la première chirurgie et le geste réalisé.

Lors de la révision, nous avons réalisé un interrogatoire des parents et enfants en âge de répondre, des photos des 2 mains posées à plat doigts écartés face palmaire et face dorsale, un examen clinique des doigts opérés et du pli inguinal.

Notre critère de jugement principal était la présence ou non d'une rétraction commissurale, quantifiée par l'échelle de Withey (18) (Fig. 3). 


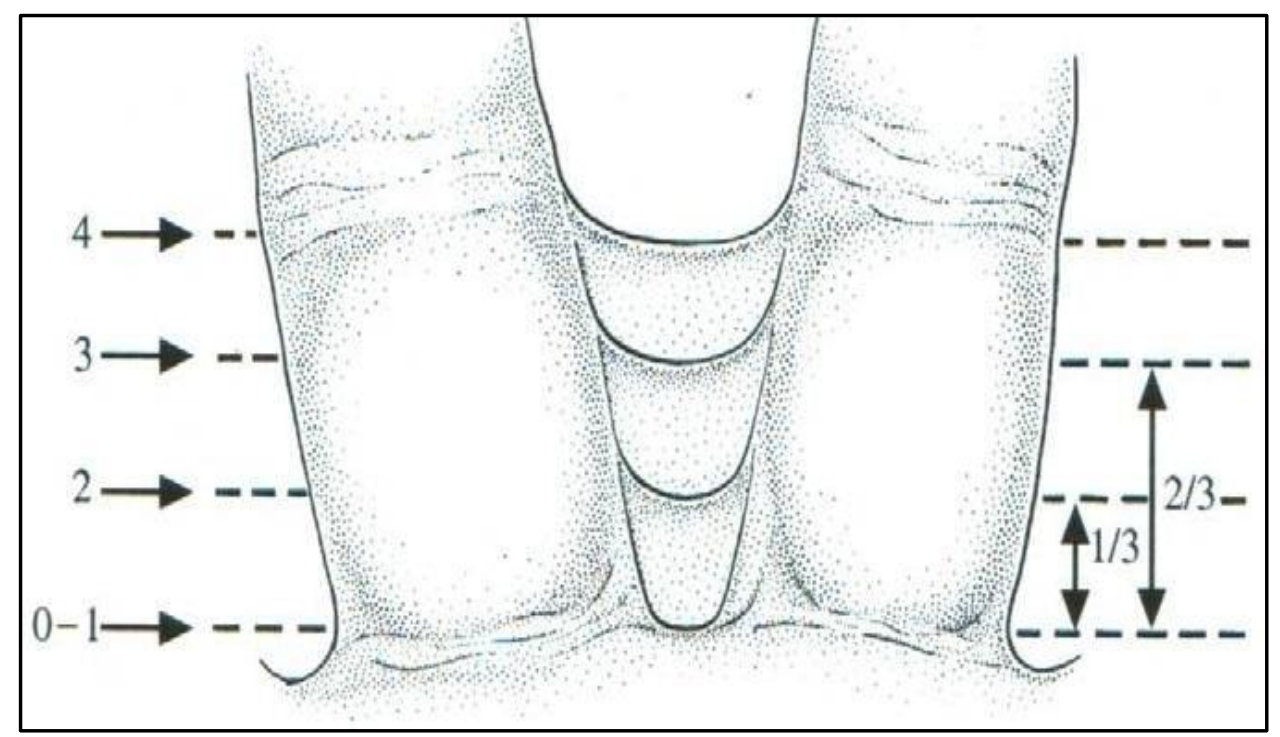

In : Withey SJ, Kangesu T, Carver N, Sommerlad BC. The open finger technique for the release of syndactyly. J Hand Surg. 2001;26(1):4-7

\begin{tabular}{|c|c|}
\hline 0 & Commissure libre, à hauteur de la commissure adjacente ou controlatérale \\
\hline 1 & Epaississement de la commissure sans web creep avec réduction de l'écartement \\
\hline 2 & Web creep résiduelle de $1 / 3$ de la première phalange \\
\hline 3 & Web creep résiduelle de $2 / 3$ de la première phalange \\
\hline 4 & Web creep résiduelle de toute la première phalange \\
\hline
\end{tabular}

Web creep : rétraction commissurale

Figure 3 : Evaluation de la rétraction commissurale selon l'échelle de Whitey (18).

Nos critères de révisions secondaires étaient : la qualité cicatricielle évaluée par l'échelle de Vancouver (Vancouver Scar Scale (VSS), Annexe 1) (19), la présence d'une pilosité et/ou d'une dyschromie au niveau des zones greffées, la mesure de l'écartement digital, la présence d'un flessum, d'une clinodactylie, l'évaluation des mobilités en flexion et extension, la sensibilité de la pulpe selon le British medical research council (BMRC) (Annexe 2) et enfin, la satisfaction en mode binaire des parents et/ou de l'enfant sur la plan fonctionnel, esthétique et global. La mesure de l'écartement digital était réalisée à partir des photos mains posées à plat et du logiciel ImageJ (Image processing and analysis in Java, National Institutes of Health, Maryland, USA) permettant de calculer un angle. 


\section{2-b. Prise en charge chirurgicale}

La chirurgie était réalisée en seul temps pour les formes bilatérales, et en deux temps pour les syndactylies atteignant deux commissures adjacentes. Si certains auteurs ont décrit des techniques permettant la prise en charge en un temps des $3^{\text {ème }}$ et $4^{\text {ème }}$ commissures d'une même main (20), notre choix d'opérer en deux temps permettait de limiter le risque vasculaire (spasme lors de la dissection) et de minimiser les tensions cutanées. L'intervention était réalisée sous anesthésie générale, avec un garrot pneumatique à la racine du membre gonflé entre 150 et $180 \mathrm{mmHg}$ et sous grossissement optique à l'aide de loupes.

Elle débutait par la réalisation d'un dessin représentant le lambeau commissural palmaire de Blauth pour la reconstruction de la néocommissure et des incisons en zigzag pour la séparation digitale (Fig. 4). Le lambeau palmaire décrit par Blauth en 1972 (14) est un lambeau en oméga oblongue ou en arche gothique prélevé au dépens de la face palmaire. Ses caractéristiques sont représentées sur la figure 5 .

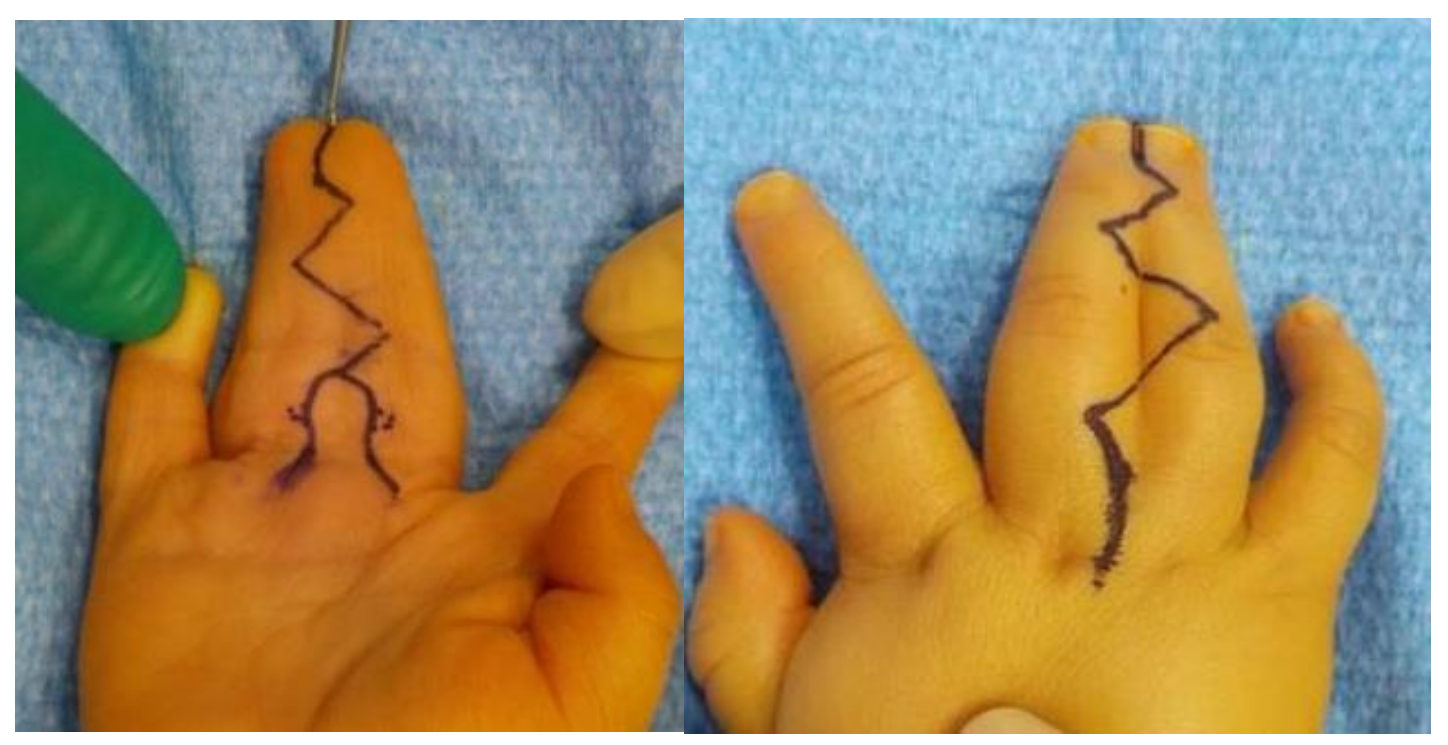

Figure 4: Dessin au stylo dermographique du lambeau palmaire selon la technique de Blauth et incisions en zig-zag. 


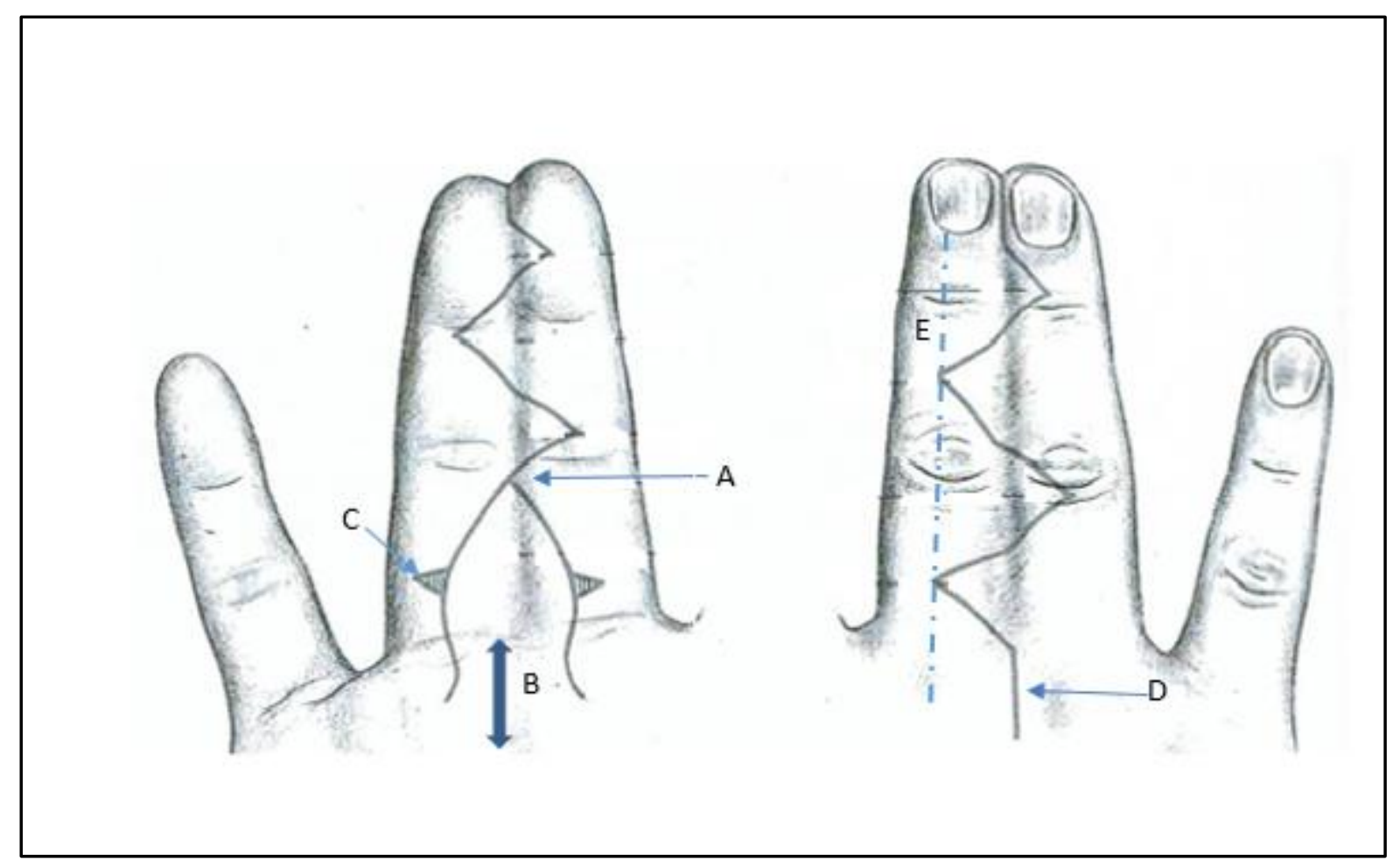

In Blauth W, Schneider-Sickert F ; Congenital Deformities of the Hand, Springer-Verlag New-York 1981.

Figure 5 : Schéma et caractéristiques géométriques du lambeau palmaire de Blauth (14).

En palmaire : La pointe du lambeau commissurale (A) s'étend à proximité du pli palmaire de l'articulation inter-phalangienne proximale. La base (B) du lambeau est placée au 2/3 distal de la distance entre le pli palmaire distal et le pli digito palmaire. A hauteur de la moitié de la première phalange, deux petits triangles latéraux (C) sont excisés permettant une meilleure mobilisation des lambeaux et évitant la formation de cicatrices longitudinales.

En dorsal : L'incision longitudinale (D) s'étend sur la hauteur de la nécommissure dorsale. Les incisions en zig-zag sont inversées entre les faces palmaire et dorsale et en largeur elles $s^{\prime}$ étendent jusqu'à la ligne médiane des doigts ( $E$ ). 
Dans les formes complètes avec un appareil unguéal commun, nous réalisions un lambeau de Buck-Gramcko (21) (Fig. 6).

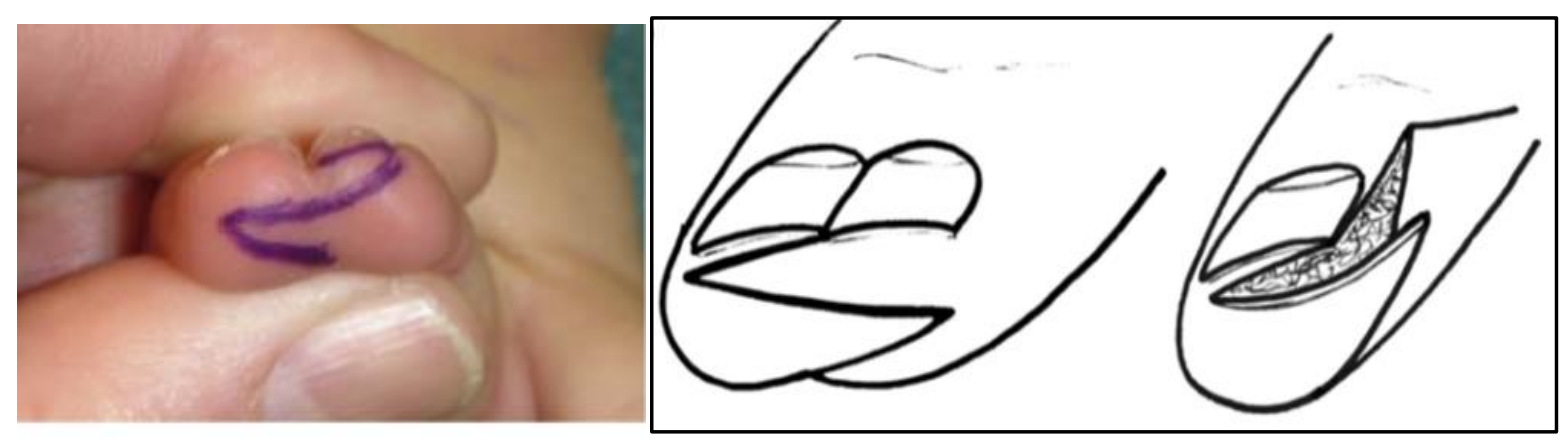

In : Samson P, Salazard B. Syndactylies. Chir Main. 2008;27:S100-14

Figure 6 : Dessin au stylo dermographique et schéma du lambeau de Buck-Gramcko (21).

La dissection des différents lambeaux s'effectuait en sous cutanée en prenant soin de ne pas léser les pédicules, la dissection au niveau de la commissure permettait de visualiser la bifurcation des pédicules artériels et nerveux (Fig. 7). Lorsqu'il existait une division basse, un des axes vasculaires était sacrifié après levée du garrot et test de clampage et en fonction de l'atteinte des commissures adjacentes. Concernant la division nerveuse, il était réalisé une intra-neurodissection.

Dans les formes complexes avec fusion osseuse de P3, il était réalisé une ostéotomie longitudinale à la lame de bistouri froid.

Les différents lambeaux étaient agencés et fixés sans tension à l'aide de fils tressés à résorption rapide (VICRYL ${ }^{\mathrm{TM}}$ RAPIDE 5/0 ou 4/0, Ethicon).

Les pertes de substances cutanées au niveau des faces latérales étaient couvertes par des greffes de peau totale dégraissées et perforées, fixées par le même fils. Les zones à greffer se situaient majoritairement au niveau de la première phalange (Fig. 8). Le prélèvement de la greffe s'effectuait au niveau du pli inguinal, la zone de prélèvement était fermée à l'aide d'un surjet de monofilament résorbable (MONOCRYL ${ }^{\mathrm{TM}} 4 / 0$, Ethicon). 


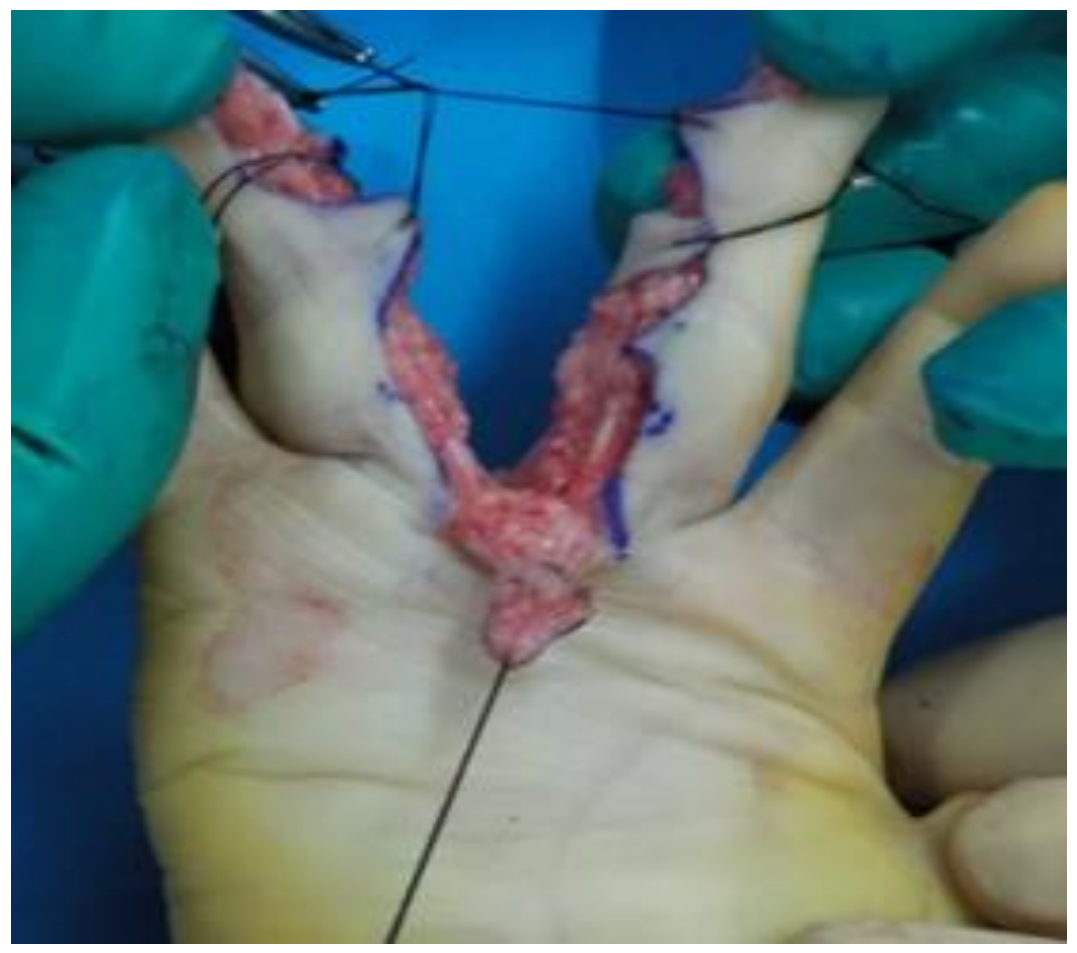

Figure 7 : Vue per opératoire des pédicules vasculo-nerveux.

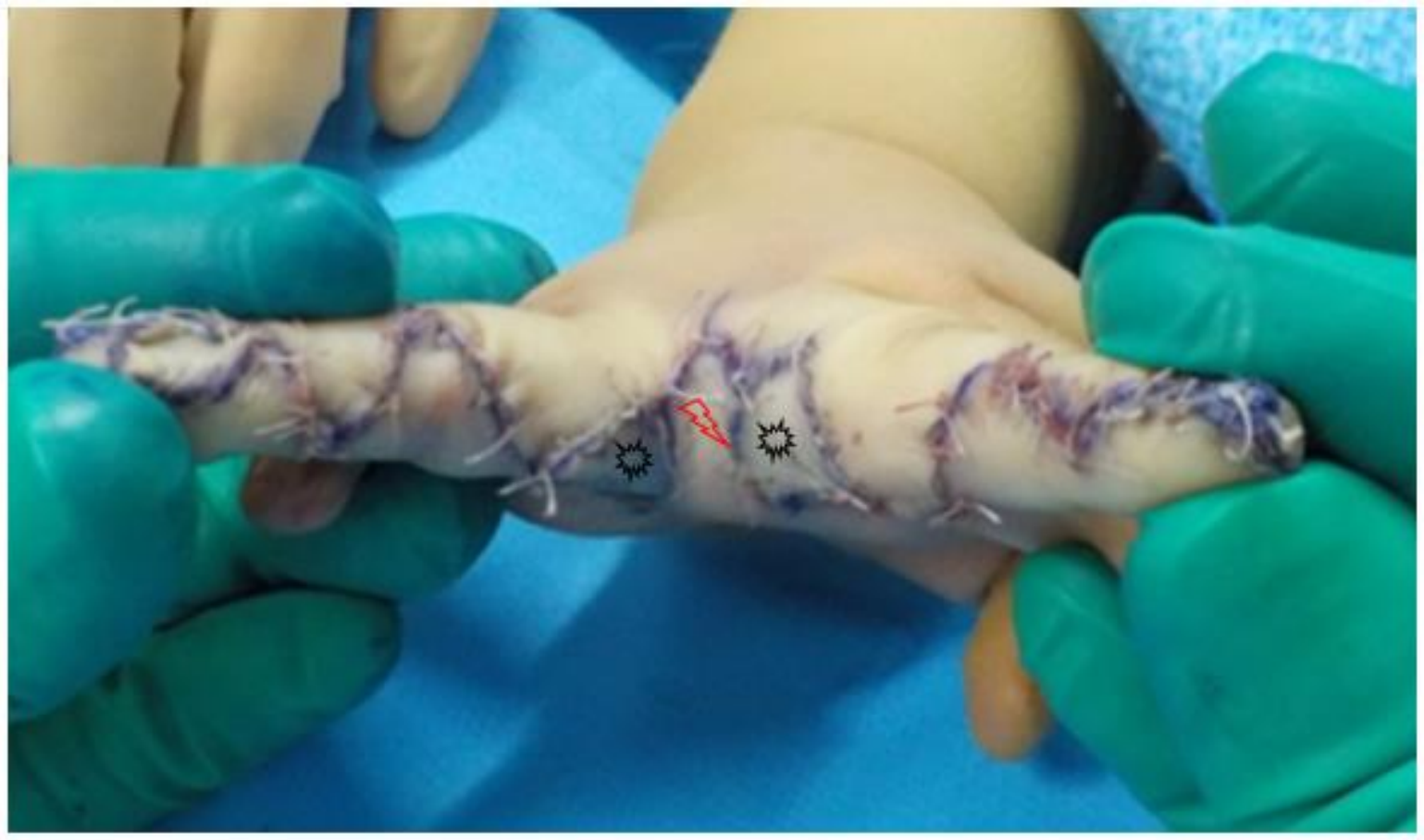

Zone de greffe de peau totale

Lambeau palmaire de Blauth

Figure 8: Vue per opératoire de l'agencement des différents lambeaux et des zones de greffes de peau totale. 
Avant la confection du pansement, le garrot était lâché afin de vérifier la vascularisation digitale et l'absence de saignements importants.

Le pansement était réalisé avec une interface grasse et l'ensemble de la main était maintenu dans une moufle pour une durée moyenne de 5 jours. La réalisation du premier pansement $s^{\prime}$ effectuait entre le $5^{\text {ème }}$ et le $7^{\text {ème }}$ jour post-opératoire et permettait le contrôle de la prise des greffes. Le suivi s'effectuait ensuite de façon hebdomadaire jusqu'à cicatrisation complète, puis de manière plus espacée sur une durée minimale de 1 an.

Lorsque cela était nécessaire une pressothérapie par cavalier en silicone était mise en place au niveau de la commissure, une fois la cicatrisation acquise.

\section{2-c. Analyses statistiques}

Les comparaisons de données à distribution normale étaient faites avec le test de Student. Le seuil de signification statistique $(p)$ était fixé à 0,05 . La recherche de dépendance ou d'indépendance des évènements était réalisée par un test du Chi 2 ou test exact de Fisher. La recherche de corrélation entre deux variables quantitatives était effectuée par le test de corrélation de Pearson. 


\section{Résultats}

\section{3-a. Population opérée}

Entre le $1^{\text {er }}$ janvier 1993 et $1^{\text {er }}$ juin 2015, 43 enfants, soit 65 commissures ont été opérés d'au moins une syndactylie syndromique ou non, simple ou complexe par la technique d'un lambeau commissural palmaire de Blauth pour la reconstruction de la commissure (Tableau 1 et Fig. 9).

\begin{tabular}{|c|c|}
\hline Caractéristiques & Cas (n) \\
\hline $\begin{array}{l}\text { Nombre enfants } \\
\text { Nombre de commissures } \\
\text { Ratio H/F }\end{array}$ & $\begin{array}{c}43 \\
65 \\
32 / 11\end{array}$ \\
\hline $\begin{array}{l}\text { SYNDACTYLIES } \\
\text { Syndromique/non syndromique } \\
\text { Formes simple/ complexe } \\
\text { Formes bilatérale }\end{array}$ & $\begin{array}{c}12 / 31 \\
48 / 17 \\
13\end{array}$ \\
\hline $\begin{array}{rr}\text { Classification Malek } & \text { A } \\
\text { B } \\
\text { C } \\
\text { DM }\end{array}$ & $\begin{array}{c}37 \\
22 \\
2 \\
4\end{array}$ \\
\hline Appareil unguéal $\begin{array}{r}\text { double } \\
\text { commun }\end{array}$ & $\begin{array}{c}63 \\
2 \\
\end{array}$ \\
\hline
\end{tabular}

DM : Données manquantes

Tableau 1: Caractéristiques de la population opérée

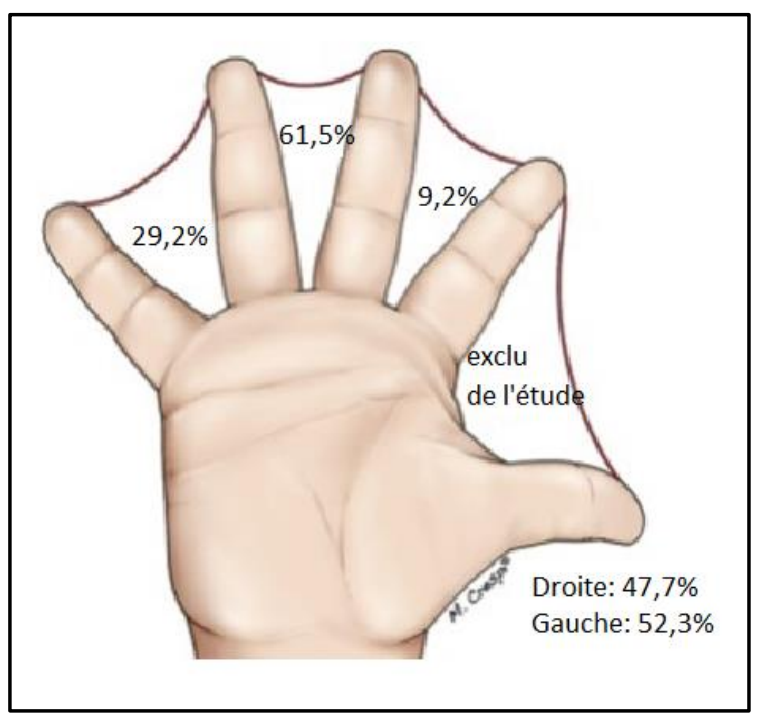

Figure 9: Répartition des commissures atteintes et latéralité de la population opérée

L'âge moyen au moment de la chirurgie était de 18,9 mois (5-73 mois). Trois anomalies per-opératoires ont été constatées: dans 2 cas il manquait un des pédicules vasculo-nerveux collatéral et dans un cas on observait l'absence d'une artère collatérale. Le délai moyen de cicatrisation était de 3,5 semaines. Dix-sept commissures (26\%) ont bénéficié de pressothérapie. 
Deux complications post-opératoires ont été notées. II s'agissait d'infections cutanées superficielles ayant régressées après soins locaux. II n'a pas été relevé d'ischémie digitale, ni de nécrose cutanée du lambeau, ni de lyse totale des greffes, ni d'hématome.

Huit commissures (12\%) ont nécessité une reprise chirurgicale pour un approfondissement ou une bride rétractile par plastie locale (plastie en Z, trident, Ostrowsky). Dans 5 cas sur les huit, il s'agissait de forme syndromique ou complexe. Le délai moyen de reprise chirurgicale était de 28,6 mois (13-65 mois).

\section{3-b. Population revue}

Nous avons revu 20 patients, soit 31 commissures. Douze patients ont été perdu de vue par absence de coordonnées ou absence de réponses et 11 ont refusé de participer à l'étude (Tableau 2 et Fig. 10).

\begin{tabular}{|c|c|}
\hline Caractéristiques & Cas (n) \\
\hline $\begin{array}{l}\text { SYNDACTYLIES } \\
\text { syndromique/non syndromique } \\
\text { simple/ complexe }\end{array}$ & $\begin{array}{c}31 \text { commissures } \\
7 / 13 \\
21 / 10\end{array}$ \\
\hline \multirow[t]{4}{*}{ Classification Malek } & 19 \\
\hline & 7 \\
\hline & 2 \\
\hline & 3 \\
\hline \multirow[t]{2}{*}{ Appareil unguéal } & 30 \\
\hline & 1 \\
\hline Age moyen lors de l'intervention & 19,3 mois \\
\hline
\end{tabular}

DM : Données manquantes

\section{Tableau 2 : Caractéristiques de la population} revue

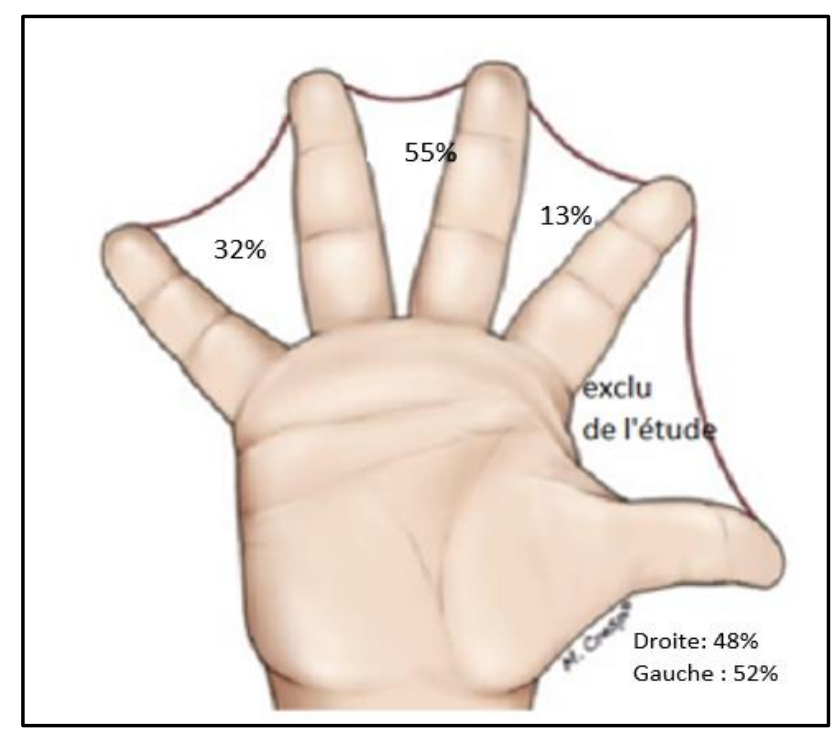

Figure 10 : Répartition des commissures atteintes et latéralité 
Le recul moyen lors de la révision était de 9 ans (11- 270 mois) et $71 \%$ des patients ont été revus avec un recul supérieur à 5 ans. L'âge moyen lors de la révision était de 10 ans et 9 mois ( 2 ans et 2 mois - 24 ans).

L'évaluation de la rétraction commissurale selon l'échelle de Withey trouvait 18 commissures (58\%) libres (grade 0), 11 commissures (36\%) avec un épaississement mais sans avancement (grade 1). Deux commissures (6\%) étaient de grade 2 et il n'y avait aucune commissure de grade 3 ou 4 (Fig. 11 et 12). Le grade moyen selon l'échelle de Withey était de 0,5 .

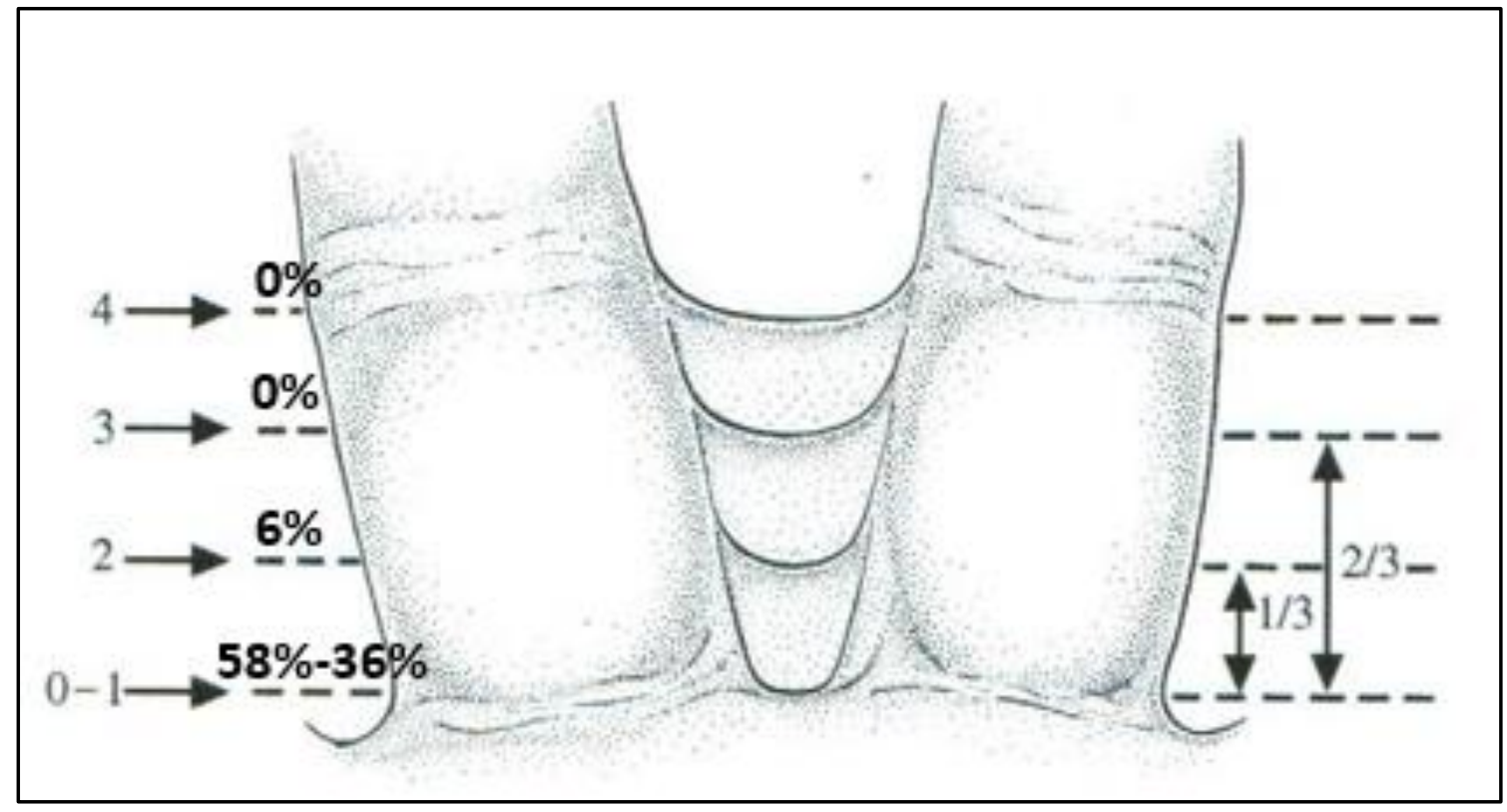

Figure 11: Répartition des commissures en pourcentage en fonction de leur grade selon l'échelle de Withey (18). 


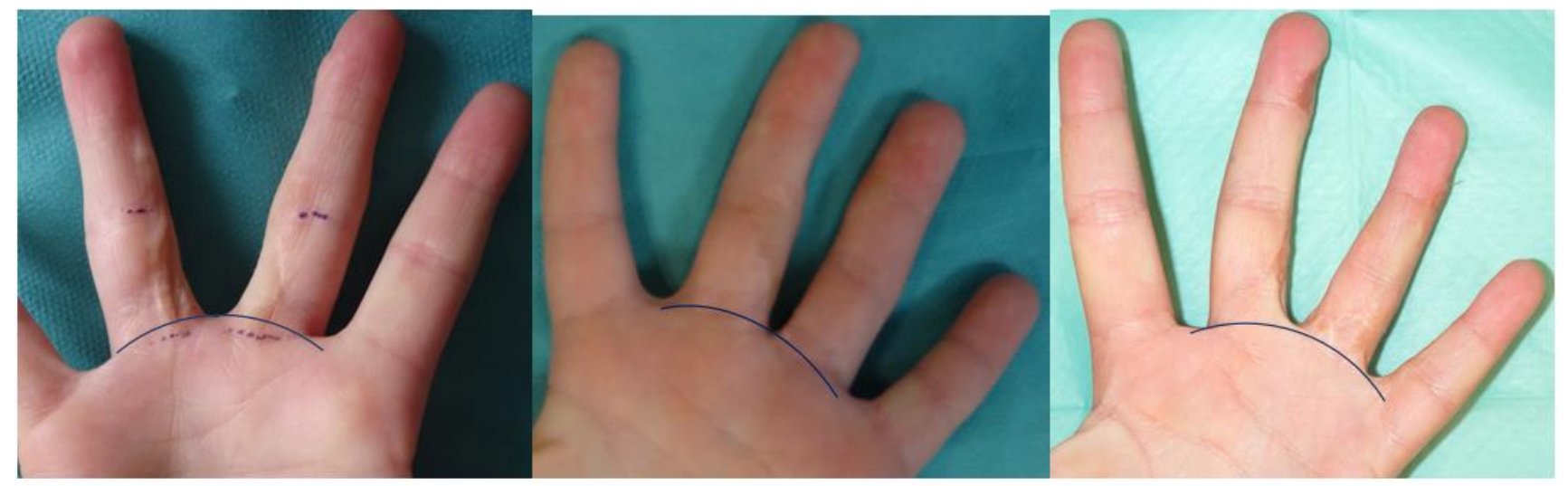

Figure 12 : Photos de $3^{\text {èmes }}$ commissures de grade 0, 1 et 2 (respectivement de gauche à droite) selon l'échelle de Withey.

Nous n'avons pas trouvé de corrélation linéaire entre l'âge au moment de la chirurgie et le stade de rétraction commissurale selon l'échelle de Withey (Fig. 13).

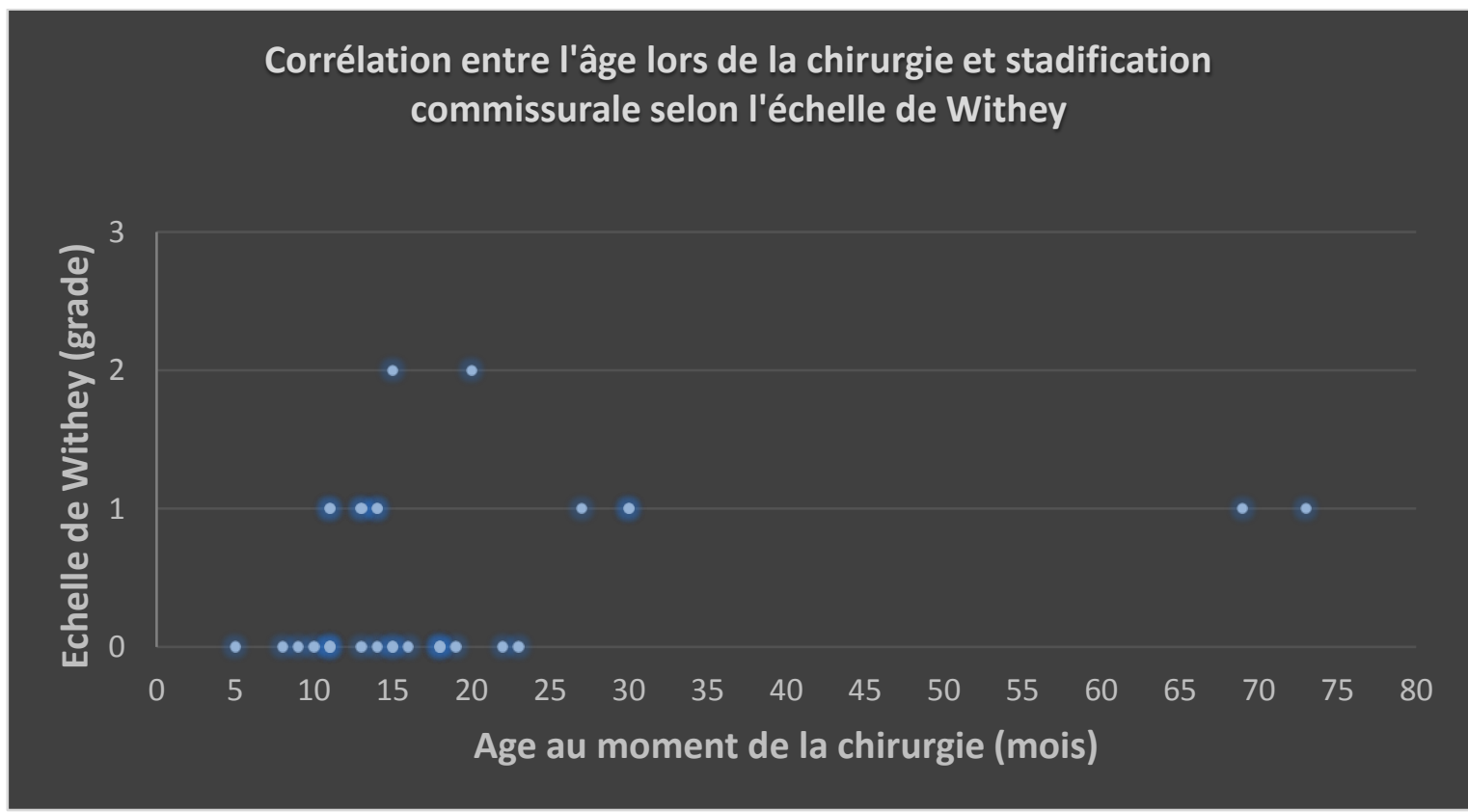

Figure 13: Graphique du grade selon l'échelle de Withey et l'âge au moment de la chirurgie 
II n’a pas était trouvé de différence statistiquement significative entre le grade de rétraction commissurale et le sexe, la commissure atteinte, les formes simple ou complexe, syndromique ou non, les formes A, B ou C selon la classification de Malek ( $p>0.05)$.

Concernant le délai de cicatrisation et le grade de la rétraction commissurale, il existait une tendance statistique $(p=0,06)$, mais par manque de puissance, nous ne pouvions pas conclure à une différence significative.

II n'y avait pas de corrélation entre le grade selon l'échelle de Withey et le délai de révision (Tableau 3).

\begin{tabular}{|c|c|c|c|c|}
\hline Critères évalués & Grade $s$ & l'échelle & Withey & \\
\hline Echelle de Withey & Grade 0 & Grade 1 & Grade 2 & \\
\hline Commissures atteintes & $\begin{array}{c}3 \\
10 \\
5 \\
\end{array}$ & $\begin{array}{l}1 \\
6 \\
4 \\
\end{array}$ & $\begin{array}{l}0 \\
1 \\
1 \\
\end{array}$ & $p 0,65$ \\
\hline Ration H/F & $13 / 5$ & $8 / 3$ & $1 / 1$ & $p 0,72$ \\
\hline Formes syndromique/non syndromique & $6 / 12$ & $6 / 5$ & $2 / 0$ & $p 0,08$ \\
\hline Formes simple/ complexe & $13 / 5$ & $6 / 5$ & $2 / 0$ & p 0,92 \\
\hline Classification Malek & $\begin{array}{c}10 \\
5 \\
0 \\
3 \\
\end{array}$ & $\begin{array}{l}7 \\
2 \\
2 \\
0 \\
\end{array}$ & $\begin{array}{l}2 \\
0 \\
0 \\
0 \\
\end{array}$ & p 0,34 \\
\hline Age moyen lors intervention (mois) & 14 & 28 & 17,5 & \\
\hline Délai moyen cicatrisation (semaine) & 3 & 3,3 & 4,5 & $p 0,06$ \\
\hline Recul moyen lors de la révision (mois) & 107 & 165 & 97 & $p 0,46$ \\
\hline
\end{tabular}

Tableau 3 : Répartition de la population en fonction du grade selon l'échelle de Withey.(18)

La valeur moyenne du VSS était de 3,6 au niveau des doigts (Tableau 4). Concernant la cicatrice au niveau de la zone de prélèvement inguinal, la valeur moyenne du VSS était de 1,2 .

Une pilosité au niveau des zones de GPT était présente dans $42 \%$ des commissures, les commissures sans pilosités étaient toujours trouvées chez des enfants de moins de 10 ans. II existait une dyschromie à type d'hyper ou d'hypo pigmentation pour 27 commissures (87\%) entre les zones greffées et les zones non greffées (Fig. 14). 


\begin{tabular}{|l|l|c|c|}
\hline \multicolumn{2}{|c|}{ Vancouver Scar Scale } & Nombre de cas & $\begin{array}{c}\text { Score } \\
\text { moyen }\end{array}$ \\
\hline Pigmentation & Normal & 3 & \\
& Hypopigmentation & 16 & 1.29 \\
& Hyperpigmentation & 12 & \\
Vascularisation & Normal & 31 & \\
& Rose & 0 & 0 \\
& Rouge & 0 & \\
\hline \multirow{5}{*}{ Elasticité } & Violet & 0 & \\
& Normal & 3 & \\
& Souple & 15 & \\
& Dur & 11 & 1,4 \\
& Bride & 2 & \\
\hline \multirow{5}{*}{ Epaisseur } & Rétraction & 0 & \\
& Normal & 6 & \\
& ] $0-2 \mathrm{~mm}[$ & 21 & 0,93 \\
& [2-mm [ & 4 & \\
\hline VSS moyen & $\geq 5 \mathrm{~mm}$ & 0 & 3,6 \\
\hline
\end{tabular}

Tableau 4 : Résultats du VSS au niveau des doigts (19)(Annexe 1).

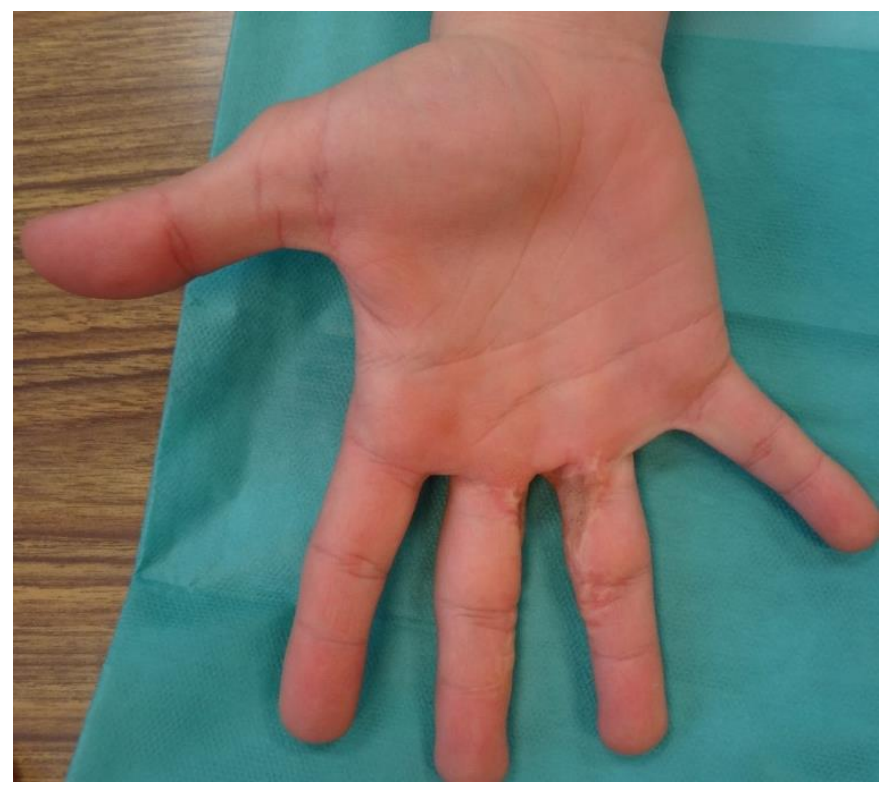

Figure 14: Patient de 21 ans pris en charge pour une syndactylie simple et complète de la $3^{\text {ème }}$ commissure droite. Présence d'une dyschromie et pilosité au niveau de la GPT (recul de 16 ans). 
L'écartement interdigital moyen au niveau des commissures opérées était de $27,6^{\circ}$. Les mobilités étaient conservées pour 20 rayons et altérées pour 11 doigts.

Dix rayons $(32,3 \%)$ présentaient un flessum au niveau des doigts adjacents à la commissure opérée, pour six d'entre eux, le flessum était réductible. Ce flessum n'était présent que pour des patients ayant des syndactylies syndromiques et complexes.

Une clinodactylie était mise en évidence pour 11 doigts (35,5\%), pour 8 d'entre eux elle était mineure (inférieure à $15^{\circ}$ ) et il s'agissait de syndactylies syndromiques dans $73 \%$ des cas.

La sensibilité de la pulpe était toujours conservée, cotée à S4 selon le BMRC.

$100 \%$ des enfants en âge de répondre étaient satisfaits sur le plan global et fonctionnel, et 92\% sur le plan esthétique. Quant aux parents, 93\% d'entre eux étaient satisfaits sur le plan global, $96,5 \%$ sur le plan fonctionnel et $83 \%$ sur le plan esthétique (Fig. 15.).

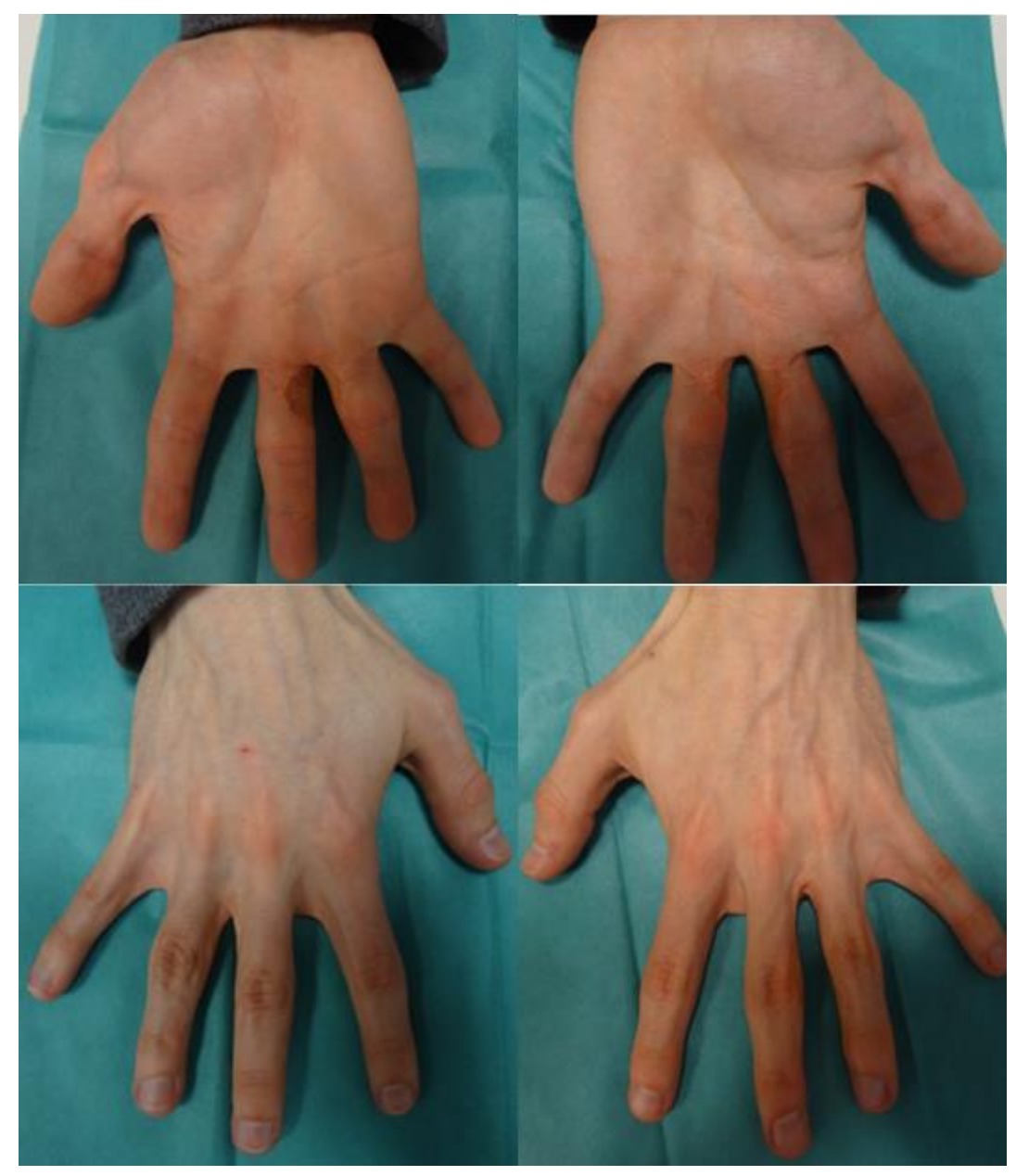

Figure 15: Résultats d'une reconstruction bilatérale de la $3^{\text {ème }}$ commissure par un lambeau palmaire de Blauth sur une vue palmaire et dorsale pour une syndactylie simple et incomplète (Malek B) (recul de 22ans). 
L'analyse de nos résultats selon l'âge, inférieur ou supérieur et égal à 18 mois au moment de la chirurgie correspondait à 2 groupes d'effectifs similaires en termes de commissures atteintes, de formes simple ou complexe. Nos résultats n'ont pas montré de différence statistiquement significative quant au délai de cicatrisation $(p=0,67)$, à la présence d'un flessum $(p=0,55)$ ou d'une clinodactylie $(p=0,18)$, à l'évaluation du VSS au niveau de la main $(p=0,53)$, à l'écartement interdigital $(p=0,86)$. La satisfaction globale des parents était de $95 \%$ dans le groupe " moins de 18 mois » et $92 \%$ dans le groupe " supérieur ou égal à 18 mois ", la satisfaction globale des enfants était de 100\% dans les 2 groupes.

L'analyse de la population en fonction de la forme simple ou complexe de la syndactylie, n'a pas identifié de différence statistiquement significative quant à la rétraction commissurale selon l'échelle de Withey $(p=0,92)$ ou l'évaluation du VSS $(p=0,07)$. Nous avons trouvé une différence statistiquement significative quant au taux de flessum $(p<0,05)$, et une mobilité plus réduite $(p<0,05)$. Les parents étaient moins satisfaits sur le plan fonctionnel $(90 \%$ vs $100 \%$ ) et esthétique ( $80 \%$ vs $86 \%$ ) dans le groupe des syndactylies complexes. 


\section{Discussion}

La reconstruction de la néocommissure constitue l'une des étapes clés dans le traitement des syndactylies congénitales et sa qualité est principalement évaluée par la présence d'une rétraction commissurale. Elle fait appel à l'utilisation d'une plastie cutanée de type lambeau local. Plus d'une quarantaine de techniques sont décrites dans la littérature depuis les 200 dernières années (22), il s'agit essentiellement de lambeaux dorsaux (15,23-26). Les lambeaux palmaires sont peu utilisés et leurs techniques peu décrites. Seules 2 études rapportent leurs résultats. En 1998, Ardouin et al. (27) ont déjà publié sur le lambeau commissural palmaire de Blauth mais leurs critères d'évaluation étaient purement subjectifs car les profondeurs commissurales été jugées comme satisfaisantes, acceptables ou mauvaises. Cependant, ils ont rapporté des résultats proches de ceux de notre série concernant l'aspect de la commissure avec seulement 2 commissures (4,3\%) jugées comme « mauvaises ». En 2010, Jose et al. (28) ont rapporté leurs résultats concernant 221 syndactylies prise en charge par un lambeau palmaire en "arche gothique » associé à des greffes de peau totale. Leur taux de rétraction commissurale était de 5,4\%. Cependant, cette étude manque de recul avec un suivi moyen de 2 ans.

Afin d'évaluer nos pratiques, nous avons quantifié la rétraction commissurale en utilisant l'échelle de Withey. Ce critère de jugement principal est objectif, clinique et reproductible. Il a toujours été évalué par le même examinateur, limitant ainsi les biais de classement. Seuls 2 commissures (6\%) étaient de grade 2 c'est-à-dire avec une rétraction commissurale se situant au niveau du premier tiers de la première phalange. Cinquante-huit pourcent des commissures étaient libres et de hauteur satisfaisante (grade 0) et $36 \%$ présentaient un épaississement commissural avec une hauteur comparable aux autres commissures (grade 1). Dans notre étude, l'âge au moment de la chirurgie, le sexe, la commissure atteinte ou la présence d'une forme complexe n'influençaient pas la rétraction commissurale de façon statistiquement significative $(p>0.05)$. Quant au délai de cicatrisation, 
le manque de puissance de l'étude ne nous a pas permis de conclure mais il existait une tendance statistique avec un délai de cicatrisation qui était plus important pour des rétractions commissurales de grade 2 .

Nous n'avons pas trouvé d'études comparatives entre l'utilisation d'un lambeau palmaire et dorsal, mais nos résultats semblent similaires à ceux de la littérature décrivant essentiellement I'utilisation de lambeaux dorsaux ou combinés (Tableau 5). Frick et al. (24) rapportent une série de 28 commissures traitées par un lambeau commissural dorsal, $75 \%$ des commissures étaient libres (grade 0 selon Withey), 14\% de grade 1, 7\% de grade 2 et une commissure de grade 3 . Bien que nous n'ayons pas constaté de commissure de grade 3 lors de notre révision ces résultats restent proches de ceux de notre série. En 2014, une série de 144 commissures montrent de bons résultats du lambeau dorsal de Flatt modifié (23) avec seulement $4 \%$ de commissures de grade 1 ou 2 mais le suivi moyen était de 5 ans et aucun enfant n'était revu en période post-pubertaire.

Les auteurs utilisant des lambeaux commissuraux dorsaux leurs attribuent comme avantages : le respect de la pente commissurale avec une reproduction de l'architecture commissurale (29), des cicatrices moins visibles car palmaires (15).

Avec le lambeau palmaire de Blauth l'architecture d'une commissure native est aussi respectée. En effet, son dessin en forme d'arche ou de feuille (leap flap selon Blauth) permet de restituer la pente commissurale et sa forme triangulaire (Fig. 4).

L'inconvénient de ce lambeau palmaire est la présence d'une cicatrice au niveau de la face dorsale de la commissure, face sociale de la main, mais cette localisation permet de placer la cicatrice en dehors des zones de tensions commissurales. En opposition, I'utilisation de lambeaux dorsaux entraine une cicatrice commissurale avec un risque de brides rétractiles palmaires. De plus, la technique du lambeau palmaire de Blauth facilite la dissection de la bifurcation des pédicules vasculo-nerveux au niveau de la commissure. 


\begin{tabular}{|c|c|c|c|c|c|c|c|c|}
\hline Séries & $\begin{array}{c}\text { Nombre de } \\
\text { commissures } \\
\text { Formes (s/c) }\end{array}$ & $\begin{array}{l}\text { Lambeau } \\
\text { commissural } \\
\text { utilisé } \\
(\mathrm{d} / \mathrm{p} / \mathrm{c})\end{array}$ & Greffe & $\begin{array}{l}\text { Age } \\
\text { moyen } \\
\text { lors de la } \\
\text { chirurgie } \\
\text { (mois) }\end{array}$ & $\begin{array}{c}\text { Recul } \\
\text { moyen } \\
\text { lors de } \\
\text { la } \\
\text { révision } \\
\text { (mois) }\end{array}$ & $\begin{array}{c}\text { Taux de } \\
\text { reprise } \\
\text { chirurgicale } \\
(\%)\end{array}$ & $\begin{array}{l}\text { Grade } \\
\text { moyen } \\
\text { de } \\
\text { Withey }\end{array}$ & $\begin{array}{c}\text { Taux de } \\
\text { rétraction } \\
\text { commissurale } \\
\text { (\%) }\end{array}$ \\
\hline 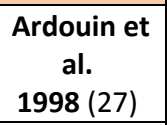 & $\begin{array}{c}47 \text { (39 revues) } \\
\text { s et c }\end{array}$ & $p$ & $\begin{array}{c}\text { GPT } \\
\text { ou CD }\end{array}$ & 24 & 36 & 10,3 & - & 5,1 \\
\hline $\begin{array}{l}\text { Withey et } \\
\text { al. } 2001 \\
(18)\end{array}$ & $\begin{array}{c}31 \\
s\end{array}$ & $d$ & $C D$ & 14 & 127 & - & 0,7 & 9,7 \\
\hline $\begin{array}{l}\text { Niranjan et } \\
\text { al. 2004 } \\
(30)\end{array}$ & $\begin{array}{c}62 \text { (25 revues) } \\
\text { s et c }\end{array}$ & $d$ & aucune & 28 & 78 & 16 & - & 16 \\
\hline $\begin{array}{l}\text { Frick et al. } \\
2008(24)\end{array}$ & $\begin{array}{c}54 \text { (28 revues) } \\
\text { s }\end{array}$ & $d$ & GPT & 13 & 70 & 9,3 & 0,4 & 10,7 \\
\hline $\begin{array}{l}\text { Lumenta et } \\
\text { al. } 2010 \\
(31)\end{array}$ & $\begin{array}{c}26 \\
s\end{array}$ & c & GPT & 52 & 137 & 11,5 & 0,6 & 7,7 \\
\hline $\begin{array}{l}\text { Jose et al. } \\
2010(28)\end{array}$ & $\begin{array}{c}221 \\
\text { s et C }\end{array}$ & p & GPT & 31 & 24 & 9 & - & 5,4 \\
\hline $\begin{array}{c}\text { Vekris et al. } \\
2010(32) \\
\end{array}$ & $\begin{array}{c}131 \\
\text { s et c }\end{array}$ & c & GPT & $<5$ ans & 137 & 15,3 & - & 7,6 \\
\hline $\begin{array}{l}\text { Hsu et al. } \\
2010(33)\end{array}$ & $\begin{array}{c}28 \\
s\end{array}$ & $d$ & aucune & 13 & 50 & 7 & - & 3,6 \\
\hline $\begin{array}{l}\text { Goldfarb et } \\
\text { al. } 2012 \\
(16)\end{array}$ & $\begin{array}{c}25 \\
c\end{array}$ & d ou c & GPT & 12 & 107 & 24 & 1,5 & 48 \\
\hline $\begin{array}{c}\text { Mallet et al. } \\
2013(25)\end{array}$ & $\begin{array}{c}59 \\
\text { s et c }\end{array}$ & $d$ & GPT & 16 & 68 & 8,4 & 0,6 & 11,9 \\
\hline $\begin{array}{c}\text { Barabas et } \\
\text { Pickford } \\
2014(23) \\
\end{array}$ & $\begin{array}{c}144 \\
\text { s et c }\end{array}$ & $d$ & GPT & 13 & 60 & 2,7 & 0,06 & 3,5 \\
\hline $\begin{array}{l}\text { Mercili et } \\
\text { al. } 2015 \\
(29)\end{array}$ & $\begin{array}{c}138 \\
\text { s et c }\end{array}$ & c & GPT & - & 30 & 7,2 & - & 8,7 \\
\hline $\begin{array}{l}\text { Truffandier } \\
\text { et al. } 2015 \\
\quad(34)\end{array}$ & $\begin{array}{c}20 \\
\text { s et c }\end{array}$ & $d$ & $\begin{array}{l}\text { Matrid } \\
\text { erm } \\
\text { avec } \\
\text { GPM }\end{array}$ & 20 & 31 & 15 & 1,2 & - \\
\hline $\begin{array}{c}\text { Karamese } \\
\text { et al. } 2016 \\
(15)\end{array}$ & $\begin{array}{c}16 \\
s\end{array}$ & c & GPT & 48 & 24 & - & 0,06 & 0 \\
\hline $\begin{array}{l}\text { Dong et al. } \\
2017 \text { (35) }\end{array}$ & $\begin{array}{c}35 \\
\mathrm{~s}\end{array}$ & $d$ & aucune & 17 & 54 & 3 & - & 5,7 \\
\hline Notre série & $\begin{array}{c}65 \text { (31 revues) } \\
\text { s et c }\end{array}$ & $p$ & GPT & 19 & 108 & 12 & 0,5 & 6 \\
\hline
\end{tabular}

$\mathrm{s}$ : simple; c : complexe

$d$ : dorsal ; $p$ : palmaire ; $c$ : combiné

$C D$ : cicatrisation dirigée

Tableau 5 : Revue de la littérature et taux de rétraction commissurale. 
Le taux de reprise chirurgicale varie de 3 à $15 \%(23,32)$ et jusqu'à $24 \%$ pour la série de Goldfarb et al. (16) qui n'étudient que des formes complexes (Tableau 5). Ces résultats montrent bien la difficulté de comparer les différentes séries de la littérature puisque les populations peuvent être très hétérogènes d'une série à l'autre en fonction du choix des auteurs d'étudier l'ensemble des syndactylies ou uniquement les formes simples. Dans notre étude, nous avons analysé les syndactylies simples, complexes et syndromiques et en effet, comme la plupart des auteurs $(23,28,32)$ nous avons constaté plus de complications en termes de flessum, et d'altération de la mobilité dans les formes complexes. Ces résultats, non surprenants, permettent d'adapter notre discours auprès des familles en les informant du risque plus important de flessum et d'altération de la mobilité chez les enfants présentant des syndactylies complexes.

En accord avec d'autres études (24,31), l'âge au moment de la chirurgie ne semblent pas avoir d'influence sur la rétraction commissurale, la qualité de la cicatrisation et la présence d'un flessum ou d'une clinodactylie. Les comparaisons entre les groupes chirurgie avant ou après 18 mois n'ont pas montré de différence statistiquement significative. Seule une étude avec un effectif plus important de patients nous permettrait d'affirmer cette hypothèse.

Un de nos critères secondaires était l'évaluation de la qualité cicatricielle par l'échelle de Vancouver (VSS). Cette échelle utilisée initialement pour les séquelles de brûlures constitue un outil complet en termes d'évaluation cicatricielle en graduant tous les aspects de la cicatrice. Concernant l'évaluation de la commissure, la vascularisation était normale dans $100 \%$ des cas, l'élasticité était normale à souple dans 57\% des cas et l'épaisseur était de moins de $2 \mathrm{~mm}$ dans $87 \%$ des cas. Ces résultats montrent une qualité cicatricielle relativement satisfaisante.

Une des autres étapes importantes dans la prise en charge des syndactylies congénitales est la couverture des faces latérales des doigts. Si l'utilisation d'un lambeau pour la reconstruction de la néocommissure est maintenant adoptée par tous, l'utilisation de greffes de peau pour la couverture des faces latérales est plus controversée.

En 2003, Deunk et al. (36) ont comparé les résultats à très long terme (suivi moyen 21 ans) entre l'utilisation de greffe de peau totale ou de greffe de peau mince pour la correction de 34 syndactylies. Leurs résultats n'ont pas permis de privilégier l'utilisation d'un type de greffe 
de peau. Les GPT présentaient plus souvent une hyperpigmentation et une pilosité. Quant aux GPM, elles étaient fréquemment instables (pelade, rougeur au froid) et un déficit de mobilité en flexion et extension était plus souvent rapporté. Un autre inconvénient lié à la GPM non évoqué dans cette publication concerne la zone de prélèvement qui est généralement douloureuse en post-opératoire et qui peut rester inesthétique.

Dans note étude, nous n'avons effectué que des GPT. Le prélèvement de la greffe s'effectuait au niveau du pli inguinal, permettant de laisser une cicatrice masquée par les sous-vêtements. Comme Deunk et al. (36), sur le plan esthétique, les complications fréquemment trouvées au niveau des zones de greffes de peau étaient la présence de pilosité et de dyschromie. La pilosité était présente dans $42 \%$ des cas dans notre série, ce chiffre étant probablement sousestimé car les commissures sans hyperpilosité n'étaient retrouvées que chez des enfants non pubères (<10 ans). Lumenta et al. (31) trouvent une pilosité dans plus de 2/3 des commissures greffées et proposent un prélèvement au niveau du tiers le plus latéral du creux inguinal. D'autres auteurs (22) préfèrent un prélèvement au niveau de la loge hypothénar pour s'affranchir d'une dyschromie et d'une pilosité mais au prix d'une cicatrice sur la bord ulnaire de la main. La peau du pli de flexion du poignet représente une alternative car c'est une zone non hormono-dépendante laissant présager l'absence de poils au moment de la puberté (9), cependant la rançon cicatricielle à l'âge adulte peut être importante. L'évaluation du VSS au niveau de la zone de prise de greffe inguinal dans notre étude montrait une qualité cicatricielle très satisfaisante (VSS moyen de 1,2).

Afin de s'affranchir des effets indésirables des greffes de peau mince (rétraction, instabilité) en conservant leurs avantages (absence de pilosité, moins pigmenté), Truffandier et al.(34) rapportent leur expérience quant à l'utilisation de derme artificiel associé à une GPM prélevée sur le cuir chevelu pour la couverture des faces latérales des doigts. Le grade moyen selon l'échelle de Withey était de 1,2 contre 0,5 trouvé dans notre série. Aucune pilosité n'était retrouvée sur les GPM mais leur recul moyen était de 2,7 ans avec des enfants tous âgés de moins de 8 ans lors de la révision. L'utilisation de derme artificiel pourrait constituer une alternative possible à la GPT mais ces résultats sont trop précoces pour être analysés.

Une autre option chirurgicale proposée par certains auteurs est de couvrir les faces latérales des doigts avec d'autres moyens locaux qu'une greffe de peau. Ces techniques 
peuvent être réalisées en utilisant deux grands principes ; l'apport d'un excès de tissu dorsal par un lambeau en ilot et/ou la pratique d'un dégraissage intensif des faces latérales des doigts (37). Deux études $(38,39)$ rapportent ces techniques mais il s'agit de séries avec un effectif et recul faibles ( $<20$ commissures et $<3$ ans), rendant leurs analyses difficilement interprétables. Niranjan et al. (30) proposent l'utilisation d'un lambeau dorsal trilobé qui permet la reconstruction de la néocommissure ainsi que la couverture des faces latérales de la base des doigts. Cela permettrait de s'affranchir de l'utilisation d'une greffe de peau sans dégraissage intensif. Dans leur étude, ils rapportaient 4 cas de rétraction commissurale (16\%) après un suivi moyen de 6,6 ans et ne trouvaient aucune nécrose de lambeau et aucun flessum. Si Sullivan et Adkinson (40) attribuent aux greffes de peau totale un rôle dans la rétraction commissurale, nous n'identifions pas plus de rétractions, seulement $6 \%$, dans notre série. $\mathrm{Ni}$ et al. (41) présentent des résultats intéressants sur un lambeau dorsal d'avancement en sablier avec dégraissage important permettant la fermeture sans utilisation de greffes de peau. Quatre-vingt-quatre pourcent des commissures de leur série étaient de bonnes hauteur et profondeur selon les critères d'Arcangelo (42), critères se rapprochant dans leur description de l'échelle de Withey, mais ils constataient la perte partielle de 2 lambeaux $(1,7 \%)$.

Ces techniques utilisant des lambeaux dorsaux d'avancement pour éviter les greffes de peau sont satisfaisantes en termes de résultats fonctionnels et esthétiques en évitant la pilosité ou l'hyperpigmentation liées aux greffes, et permettent un gain de temps chirurgical. Cependant, elles sont difficilement réalisables chez des enfants présentant des syndactylies multiples et responsables d'importantes cicatrices au niveau de la face dorsale de la main. De plus, et l'étude de Ni et al. (41) le confirme, il existe un risque de nécrose cutanée du lambeau en cas de dégraissage agressif.

Pour les patients présentant une gêne essentiellement esthétique du fait d'une pilosité ou d'une pigmentation trop importante, il serait possible de proposer secondairement une thérapie par laser avec de bons résultats (43).

Notre étude présente des limites par son caractère rétrospectif et le nombre d'enfants perdus de vue mais nos résultats sont satisfaisants et notre recul fait partie des plus importants. 


\section{Conclusion}

La qualité de la prise en charge de la syndactylie embryonnaire repose essentiellement sur l'absence de rétraction commissurale. La reconstruction de la commissure est largement privilégiée, dans la littérature et en pratique, par l'utilisation d'un lambeau d'origine dorsale. Notre étude montre que le prélèvement d'un lambeau palmaire selon la technique de Blauth offre des résultats tout aussi satisfaisants quant au taux de rétraction commissurale ( $6 \%$ de grade 2 selon l'échelle de Withey). Par ailleurs, il s'agit d'un lambeau fiable et facilitant la dissection au niveau de la bifurcation artérielle et nerveuse de la commissure. Il permet la restitution de la pente commissurale dorsale en évitant le risque de brides rétractiles palmaires. Nos résultats fonctionnels les moins bons sont plutôt liés aux syndactylies de forme complexe responsables de flessum et d'une perte de mobilité plus importante. Enfin, les résultats plus décevants mais d'ordre esthétique de notre série sont liés aux caractéristiques de la greffe de peau totale (pilosité et dyschromie), et ceci nous amène à réfléchir sur nos pratiques quant à la localisation de son prélèvement ou de modifications techniques. 


\section{Bibliographie}

1. Lovell WW, Winter RB. Lovell and Winter's Pediatric Orthopaedics. Lippincott Williams \& Wilkins; 2014.

2. Perrin J, Geoffroy-Siraudin C, Metzler-Guillemain C. La main : embryologie et principaux mécanismes malformatifs. Chir Main. 2008;27:S2-6.

3. Schwabe G, Mundlos S. Genetics of Congenital Hand Anomalies. Handchir Mikrochir Plast Chir. 2004;36(02/03):85-97.

4. Chen C-P, Lin C-J, Chen Y-N, Chern S-R, Chen S-W, Lai S-T, et al. Molecular genetic characterization of a prenatally detected de novo interstitial deletion of chromosome $2 q$ (2q31.1-q32.1) encompassing HOXD13 , ZNF385B and ZNF804A associated with syndactyly and increased first-trimester nuchal translucency. Taiwan J Obstet Gynecol. 2017;56(3):398-401.

5. Man LX, Chang B. Maternal cigarette smoking during pregnancy increases the risk of having a child with a congenital digital anomaly. Plast Reconstr Surg. 2006;117(1):301-08.

6. Swanson AB. A classification for congenital limb malformations. J Hand Surg. $1976 ; 1(1): 8-22$.

7. De Smet L, IFSSH. International Federation for Societies for Surgery of the Hand JSSH. Japanese Society for Surgery of the Hand. Classification for congenital anomalies of the hand: the IFSSH classification and the JSSH modification. Genet Couns Geneva Switz. 2002;13(3):331-8.

8. Tonkin MA, Tolerton SK, Quick TJ, Harvey I, Lawson RD, Smith NC, et al. Classification of congenital anomalies of the hand and upper limb: development and assessment of a new system. J Hand Surg. 2013;38(9):1845-53.

9. James I. Syndactylie embryonnaire. In: Captier G, Galinier P, Picard A Chirurgie plastique de l'enfant et de l'adolescent. $2015^{e}$ éd. Sauramps medical;

10. Castilla EE, Paz JE, Orioli-Parreiras IM, Opitz JM, Hermann J. Syndactyly: frequency of specific types. Am J Med Genet A. 1980;5(4):357-64.

11. Tonkin MA. Failure of Differentiation Part I: Syndactyly. Hand Clin. mai 2009;25(2):171-93. 
12. Loréa $P$, Coessens $B$. Evolution of surgical techniques for skin releases in the treatment of simple congenital syndactyly: a review. Eur J Plast Surg. 2001;24(6):275-81.

13. Samson P, Salazard B. Syndactylies. Chir Main. 2008;27:S100-14.

14. Blauth W. Syndaktylien der Hand.Dtsch Arztebl. 1972;(69):2012-21.

15. Karamese M, Akdag O, Selimoglu MN, Unal Yıldıran G, Tosun Z. V-Y and rectangular flap combination for syndactyly repair. J Plast Surg Hand Surg. 2016;50(2):102-6.

16. Goldfarb CA, Steffen JA, Stutz CM. Complex Syndactyly: Aesthetic and Objective Outcomes. J Hand Surg. 2012;37(10):2068-73.

17. Malek R. Syndactylies congénitales. In: Traité de chirurgie de la main. Masson. Paris: Tubiana R; 1998. 326-38.

18. Withey SJ, Kangesu T, Carver N, Sommerlad BC. The open finger technique for the release of syndactyly. J Hand Surg. 2001;26(1):4-7.

19. Baryza MJ, Baryza GA. The Vancouver Scar Scale: an administration tool and its interrater reliability. J Burn Care Rehabil. 1995;16(5):535-8.

20. Tian X, Xiao J, Li T, Chen W, Lin Q, Chim H. Single-Stage Separation of 3- and 4-Finger Incomplete Simple Syndactyly With Contiguous Gull Wing Flaps: A Technique to Minimize or Avoid Skin Grafting. J Hand Surg. 2017;42(4):257-64.

21. Buck-Gramcko, D. Congenital malformations. In: Nigst, H, Buck-Gramcko, D, Millesi, H, Lister, GD. Hand surgery. New York: Thieme; 1988. Vol. 1:12.22-12.23.

22. Hutchinson DT, Frenzen SW. Digital Syndactyly Release: Tech Hand Up Extrem Surg. 2010;14(1):33-7.

23. Barabás AG, Pickford MA. Results of syndactyly release using a modification of the Flatt technique. J Hand Surg Eur Vol. 2014;39(9):984-988.

24. Frick $L$, Fraisse $B$, Wavreille $G$, Fron $D$, Martinot V. Résultats du traitement chirurgical des syndactylies simples par lambeau commissural dorsal. À propos de 54 commissuroplasties. Chir Main. 2008;27(2-3):76-82.

25. Mallet $C$, Ilharreborde B, Jehanno P, Litzelmann E, Valenti P, Mazda K, et al. Comparative study of 2 commissural dorsal flap techniques for the treatment of congenital syndactyly. J Pediatr Orthop. 2013;33(2):197-204.

26. Glicenstein J. Les syndactylies congénitales. Cah D’enseignement Société Fr Chir Main. 1990;2:95-11. 
27. Ardouin T, Poirier P, Dabos N, Rogez J-M. Traitement des syndactylies par lambeau commissural palmaire selon Blauth. La Main. 1998;3:385-92.

28. Jose RM, Timoney N, Vidyadharan R, Lester R. Syndactyly correction: an aesthetic reconstruction. J Hand Surg Eur Vol. 2010;35(6):446-450.

29. Mericli AF, Black JS, Morgan RF. Syndactyly Web Space Reconstruction Using the Tapered M-to-V Flap: A Single-Surgeon, 30-Year Experience. J Hand Surg. 2015;40(9):1755-63.

30. Niranjan NS, Azad SM, Fleming ANM, Liew SH. Long-term results of primary syndactyly correction by the trilobed flap technique. Br J Plast Surg. 2005;58(1):14-21.

31. Lumenta DB, Kitzinger HB, Beck H, Frey M. Long-Term Outcomes of Web Creep, Scar Quality, and Function After Simple Syndactyly Surgical Treatment. J Hand Surg. 2010;35(8):1323-9.

32. Vekris MD, Lykissas MG, Soucacos PN, Korompilias AV, Beris AE. Congenital syndactyly: outcome of surgical treatment in 131 webs. Tech Hand Up Extrem Surg. 2010;14(1):2-7.

33. Hsu VM, Smartt JM, Chang B. The Modified V-Y Dorsal Metacarpal Flap for Repair of Syndactyly without Skin Graft: Plast Reconstr Surg. 2010;125(1):225-32.

34. Truffandier $M-V$, Perrot $P$, Duteille $F$. Intérêt d'un substitut dermique en 1 temps (Matriderm (C) dans la couverture des pertes de substance de la face latérale des doigts longs lors d'une cure de syndactylie congénitale : à propos de 20 commissures. Ann Chir Plast Esthét. 2015;60(4):284-90.

35. Dong $Y$, Wang $Y$. The use of a dorsal double-wing flap without skin grafts for congenital syndactyly treatment: A STROBE compliant study. Medicine (Baltimore). 2017;96(30):e7639.

36. Deunk J, Nicolai JPA, Hamburg SM. Long-term results of syndactyly correction: fullthickness versus split-thickness skin grafts. J Hand Surg. 2003;28(2):125-130.

37. Braun T, Trost J, Pederson W. Syndactyly Release. Semin Plast Surg. 2016;30(04):162-70.

38. Sharma RK, Tuli P, Makkar SS, Parashar A. End-of-Skin Grafts in Syndactyly Release: Description of a New Flap for Web Space Resurfacing and Primary Closure of Finger Defects. HAND. 2009;4(1):29-34.

39. Aydn A, Ozden BC. Dorsal Metacarpal Island Flap in Syndactyly Treatment: Ann Plast Surg. 2004;52(1):43-8. 
40. Sullivan MA, Adkinson JM. A Systematic Review and Comparison of Outcomes Following Simple Syndactyly Reconstruction With Skin Grafts or a Dorsal Metacarpal Advancement Flap. J Hand Surg. 2017;42(1):34-40.

41. Ni F, Mao H, Yang X, Zhou S, Jiang Y, Wang B. The Use of an Hourglass Dorsal Advancement Flap Without Skin Graft for Congenital Syndactyly. J Hand Surg. 2015;40(9):1748-54.

42. D'Arcangelo M, Gilbert A, Pirrello R. Correction of syndactyly using a dorsal omega flap and two lateral and volar flaps. A long-term review. J Hand Surg Edinb Scotl. 1996;21(3):320-4.

43. Mishra A, Nelson K, McNally S, Gorst C, McArthur P. Laser ablation of abnormal skin pigmentation post syndactyly release. J Plast Reconstr Aesthet Surg. 2010;63(10):1753-5. 


\section{Annexes}

\section{Annexe 1: Vancouver Scar Scale}

\begin{tabular}{|lll|}
\hline Vancouver Scar Scale & & \\
\hline Pigmentation & Normal & 0 \\
& Hypopigmentation & 1 \\
& Hyperpigmentation & 2 \\
\hline \multirow{3}{*}{ Vascularisation } & Normal & \\
& Rose & 0 \\
& Rouge & 1 \\
& Violet & 2 \\
Elasticité & & 3 \\
& Normal & \\
& Souple & 0 \\
& Dur & 1 \\
& Bride & 2 \\
& rétraction & 3 \\
& & 4 \\
\hline \multirow{3}{*}{ Epaisseur } & Normal & \\
& ] 0-2 mm [ & 0 \\
& [2-mm [ & 2 \\
& $\geq 5 \mathrm{~mm}$ & 3 \\
\hline
\end{tabular}


Annexe 2: British Medical Research Council

\section{Échelle sensitive (British Medical Research Council)}

S4 : sensibilité normale; distance de discrimination entre 2 points de $15 \mathrm{~mm}$ ou moins ; reconnaissance tactile.

$\mathrm{S} 3+$ : récupération de la discrimination entre 2 points dans le territoire autonome du nerf.

S3 : récupération de la sensibilité tactile et douloureuse superficielle dans la totalité du territoire autonome du nerf avec disparition de toute hypersensibilité antérieure.

S2 : récupération partielle de la sensibilité tactile et douloureuse superficielle dans le territoire autonome du nerf.

S1: récupération de la sensibilité douloureuse profonde dans le territoire autonome du nerf.

S0 : absence de sensibilité dans le territoire autonome du nerf. 
Évaluation du lambeau palmaire de Blauth lors de la prise en charge des syndactylies congénitales : à propos de 31 commissures

\section{RESUME :}

Introduction: Les syndactylies congénitales résultent d'un défaut de différenciation tissulaire entrainant l'absence de séparation digitale au cours de l'embryogénèse. Il s'agit d'une malformation fréquemment rencontrée dont la prise en charge chirurgicale est décrite depuis le XIX ${ }^{\text {ème }}$ siècle. Elles sont de sévérité variable et peuvent être isolées ou associées à d'autres anomalies dans le cadre de syndromes malformatifs. La construction de la néocommissure fait partie des éléments déterminants de la qualité du traitement chirurgical. L'objectif principal de notre étude ćtait de quantifier la rétraction commissurale lors de la prise en charge des syndactylies congénitales opérées par un lambeau palmaire de Blauth.

Matériels et méthodes: Nous avons réalisé une étude monocentrique rétrospective. Nous avons inclus les enfants présentant au moins une syndactylie des doigts longs, simple ou complexe, syndromique ou non, opérés entre le $1^{\text {er }}$ janvier 1993 et le $1^{\mathrm{er}}$ juin 2015 par une plastie commissurale palmaire selon la technique de Blauth. Pour compléter la couverture des faces latérales des doigts, tous les enfants bénéficiaient d'une greffe de peau totale prélevée au niveau du pli inguinal. Nous avons recueilli les données épidémiologiques et de prise en charge à partir des dossiers, puis nous avons évalué les enfants lors d'une consultation de révision. Notre critère de jugement principal était la rétraction commissurale évaluée selon l'échelle de Withey. Nos critères de jugements secondaires étaient: la qualité cicatricielle selon l'échelle de Vancouver, la présence d'une pilosité et/ou d'une dyschromie, la mesure de l'écartement digital, la présence d'un flessum digital, d'une clinodactylie, la mesure de la mobilité en flexion et en extension, la sensibilité de la pulpe et la satisfaction des parents et/ou de l'enfant.

Résultats : Soixante-cinq commissures ont été opérées avec un âge moyen lors de la chirurgie de 18,9 mois. Huit commissures ont nécessité une reprise chirurgicale. 
Nous avons revu 20 patients soit 31 commissures, avec un recul moyen de 9 ans. Selon la classification de Withey, $58 \%$ des commissures étaient évaluées de grade $0,36 \%$ de grade 1 et $6 \%$ de grade 2 . Aucune commissure n'était quantifiée de grade 3 ou 4 . L'âge au moment de la chirurgie, le sexe, la commissure atteinte, le type de la syndactylie n'étaient pas corrélés à la présence d'une rétraction commissurale. Une diminution de la mobilité et la présence d'un flessum étaient plus souvent présentes dans les formes complexes. Quatre-vingt-sept pourcent des greffes de peau totale présentaient une dyschromie et $42 \%$ une pilosité. Sur le plan global, $93 \%$ des parents étaient satisfaits et $100 \%$ des enfants en âge de répondre.

Conclusion: Le lambeau palmaire de Blauth est un lambeau fiable assurant de bons résultats fonctionnels et un taux de rétraction commissurale faible pour la reconstruction de la néocommissure. Sur le plan esthétique les plaintes principales concernent la dyschromie et la pilosité liées aux greffes de peau totale.

(*) VU ET PERMIS D'IMPRIMER

(*) Grenoble, le 22/12/17

(*) LE DOYEN

$(*)$ P. MORAND

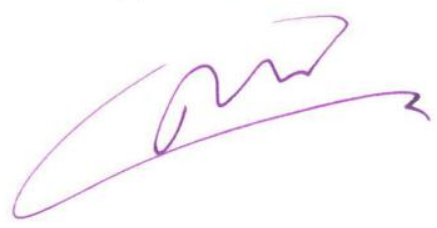

(*) PROFESSEUR Françon MouteT

CHRU DE GRENOBLE

Chirurgie de la Main et des Brulés

13 DEC. 2017

PrF. MOUTET 